\title{
GIOVAN BATTISTA MORGAGNI E IL DIBATTITO FRA MEDICINA RAZIONALE E MEDICINA EMPIRICA
}

\author{
FABIO ZAMPIERI (*)
}

SunTO. - Giovanni Battista Morgagni è considerato il padre dell'anatomia patologica. Il suo contributo può essere contestualizzato nell'ambito dello straordinario sviluppo dell'anatomia fra XVII e XVIII secolo, periodo nel quale questa disciplina divenne la regina delle scienze della natura. Una nuova patologia basata sull'anatomia fu possibile grazie alla prospettiva meccanicistica che aveva caratterizzato questa scienza nel XVII secolo, in particolare con l'opera di Marcello Malpighi, che Morgagni considerò come un maestro. L'approccio di Malpighi e degli altri 'iatromeccanici' fu oggetto di un ampio dibattito nel quale furono opposti e intrecciati i fautori del meccanicismo e dell'empirismo e i sostenitori della medicina antica, cioè ippocratico-galenica, e di quella moderna, cioè 'neoterica'. Il metodo anatomo-clinico di Morgagni può essere compreso appieno solo contestualizzato all'interno di questa controversia.

$* * *$

ABSTRACT. - Giovanni Battista Morgagni is considered the father of pathological anatomy. His contribution can be contextualized in the extraordinary development of anatomy between the seventeenth and the eighteenth centuries, because along this period anatomy became the most important among the natural sciences. A new pathology based on anatomy was possible thanks to the mechanistic perspective which characterized this science during the seventeenth century, in particular through the work of Marcello Malpighi, whom Morgagni considered as his master. The approach of Malpighi and other 'iatromechanists' was widely debated: supporters of mechanisms and empiricism, as well as supporters of the Ancients, or Hippocratic-Galenic medicine, and of the Moderns, or 'Neoteric' medicine, were opposed and interlaced. The anatomo-clinical method of Morgagni can be fully understood only by being contextualized within this debate.

(*) Università degli Studi di Padova, Italia. E-mail: fabio.zampieri@unipd.it 


\section{INTRODUZIONE: MORGAGNI E L'ANATOMIA PRATICA}

Giovanni Battista Morgagni (1682-1771), nato a Forlì, laureato in medicina a Bologna, docente di Medicina Teorica (1711-1715) e di Anatomia (1715-1771) a Padova ${ }^{1}$, è universalmente noto come padre dell'anatomia patologica, disciplina che si considera sistematicamente fondata col suo celeberrimo De sedibus et causis morborum per anatomen indagatis del $1761^{2}$.

Si ritiene che l'anatomia patologica sia stata, proprio a partire dal contributo morgagnano, la disciplina che più di tutte favorì quella frattura epistemologica fondamentale che portò alla medicina moderna, determinando l'abbandono definitivo del paradigma umorale che dominava la teoria e la pratica medica sin dall'epoca classica ${ }^{3}$. Secondo il modello umorale, infatti, le malattie erano causate dallo squilibrio fra i quattro umori che si ipotizzavano alla base del funzionamento del corpo, ovverosia sangue, flemma, bile nera e bile gialla. Sebbene la dinamica degli umori potesse essere inquadrata anche secondo un certo schema anatomico, la concezione patologica che ne derivava non implicava una stretta e sistematica correlazione fra danno organico e sintomo clinico e, di conseguenza, l'indagine autoptica non comportava alcun valore diagnostico, né la possibilità di una maggiore conoscenza dei meccanismi fisiopatologici. Già il titolo stesso dell'opera di Morgagni, invece, sembrava porsi in una prospettiva del tutto nuova: l'anatomia, cioè, permetteva di indagare non solo le «sedi», ma anche le «cause» delle malattie, proprio perché sede e struttura del danno organico erano intimamente correlate con i processi morbosi e i sintomi clinici

1 F. Zampieri, Il metodo anatomo-clinico fra meccanicismo ed empirisimo. Marcello Malpighi, Antonio Maria Valsalva e Giovanni Battista Morgagni, Roma, L'Erma di Bretschneider, 2016.

2 G. B. Morgagni, De sedibus et causis morborum per anatomen indagatis, Venetiis, Typographia Remondiniana, 1761. Nel corso di questo saggio, pur riferendoci alla suddivisione originale del testo morgagnano, citeremo i brani del De sedibus dalla seguente traduzione italiana: P. Maggesi (a cura di), Delle sedi e delle cause delle malattie anatomicamente investigate da Gio. B. Morgagni, 15 voll., Milano, Felice Rusconi, 18231828. Con questa eccezione, le traduzioni in italiano dei brani tratti da opere in altre lingue sono a cura di chi scrive.

3 M.D. Grmek, Morgagni e la scuola anatomo-clinica di Parigi, in V. Cappelletti e F. Di Trocchio (a cura di), De sedibus, et causis. Morgagni nel centenario, Roma, Istituto della Enciclopedia Italiana, 1986, pp. 173-184. 
che ne derivavano.

Questa breve ricostruzione, per quanto corretta in alcune delle sue linee essenziali, è, allo stesso tempo, fuorviante per diverse ragioni. Da una parte, infatti, essa si basa su un'eccessiva semplificazione del modello umorale che non tiene conto del ruolo fondamentale che l'anatomia ebbe nella sua elaborazione e nel suo sviluppo nel corso dei secoli. D'altra parte, Morgagni viene posto come il punto di partenza di un nuovo modo di fare e concepire la medicina, il che non permette di cogliere quanto la sua opera possa essere considerata, piuttosto, come il punto di arrivo e, in un certo modo, l'ultima espressione di una tradizione medica secolare che, proprio in quanto tale, liberò quanto potremmo definire un nuovo territorio epistemologico, il quale verrà colonizzato solo dagli autori a lui successivi. Morgagni, infatti, può essere visto come l'ultimo esponente del dibattito fra medicina 'razionale' e medicina 'empirica' - il punto principale del quale era, appunto, il ruolo dell'anatomia - che aveva caratterizzato lo sviluppo della medicina sin dalla scuola alessandrina del III secolo a.C. e come colui che, finalmente, riuscì a risolverlo. A questo dibattito e al ruolo di Morgagni ci concentreremo nel corso di questo saggio.

Potremmo sostenere, in modo in parte provocatorio, che Morgagni, innanzitutto, non sia stato il padre dell'anatomia patologica. L'anatomia patologica come disciplina istituzionalizzata si affermerà, infatti, solo qualche decennio successivo rispetto al suo De sedibus, in particolare presso la scuola anatomo-clinica francese e presso la scuola medica viennese ${ }^{4}$, mentre il primo testo di anatomia patologica in senso stretto è considerato il The Morbid Anatomy del medico inglese Matthew Baille (1761-1823) del 17935.

Morgagni, in effetti, considerava la sua opera maggiore come un testo di «anatomia medica» o «anatomia pratica» e, con ciò, si richiamava a una lunghissima tradizione che risaliva fino alla medicina classica. A questo riguardo, vale la pena riportare una lunga citazione del De sedibus:

Di fatto, allorché nei remotissimi tempi non era permesso di notomizzare i cadaveri umani, Ippocrate, o coloro che gli succedettero dappres-

4 F. Zampieri, Da Morgagni alla patologia molecolare. Teorie e modelli dell'anatomia patologica, Padova, Libraria Padovana Editrice, 2012, pp. 63-136.

5 M. Baille, The morbid anatomy of some of the most important parts of the buman body, London, J. Johnson, 1793. 
so, ricercarono le sedi e le cause delle malattie nelle interiora dei bruti, come rilevasi dagli antichi libri di questo autore, o da quelli che nel numero degl'ippocratici scritti ripongonsi. Un tal costume fu conservato da Galeno e da altri prima e dopo di lui, e di sovente anche in tempi assai recenti; e parimente alla giornata volentieri si segue, allorché se ne offre l'occasione ... Dal che ne avvenne che, o prima o dopo Galeno, in somma sino a che fu concesso di dissecare cadaveri umani, si accolse con ardore la facoltà, d'altra parte rarissima, secondo i luoghi e le circostanze, d'incider questi ultimi ... Ma dall'istante che questa permissione incominciò finalmente ad aver luogo in Italia, e a divenir per gradi vie più frequente, è provato dalle opere che comparvero avanti il XV secolo, o dopo il principio del secolo seguente, per esempio da quelle di Alessandro Benedetti, e soprattutto di Antonio Benivieni ... Ma dopo costoro, che tra gl'Italiani per primi nominai, s'incominciarono ad aprire più di sovente i corpi umani presso le altre nazioni incivilite ... sino agli autori più moderni ... che, parlando delle malattie e della cura delle medesime, hanno riferito quello che col mezzo dell'anatomia si suol rinvenire nelle singole morbose affezioni ... A niuno può essere ignoto, ancorché abbia soltanto esaminato il principio del secondo esercizio della circolazione del sangue di Arveo, a quante dissezioni di corpi morbosi debba quest'uomo incomparabile quello ch'esso chiamava Anatomia medica, e di quanta utilità egli pensava che dovess'essere alla medicina ... non ti abbia a recar meraviglia s'io riputai non indegno ... il mio progetto d'insistere nelle ricerche, che i maestri dell'arte salutare fatte non avrebbero in ogni tempo e luogo con ogni possibile sforzo, se quelle non fossero state della più grande utilità ${ }^{6}$.

Come si evince da questo lungo passo, la pratica di ricercare la «sede e le cause» delle malattie nell'anatomia del corpo dell'uomo - o degli animali, qualora la dissezione umana non fosse consentita - risaliva, secondo Morgagni, sino agli albori della medicina occidentale. Per quanto riguarda i tempi più antichi, Morgagni cita, per esempio, gli «ippocratici scritti» e Galeno. In relazione al corpus ippocratico, l'affermazione potrebbe sembrare piuttosto sorprendente, visto che all'anatomia, di per sé, non si rivolge un'attenzione particolare nell'ampia gamma di scritti ricondotti alla scuola del celebre medico greco del $\mathrm{V}$ secolo a.C. 7 . Tuttavia, esistono svariati passaggi, sparsi in diversi trattati,

6 G. B. Morgagni, De sedibus et causis morborum per anatomen indagatis, cit., Prefazione al Libro Secondo.

7 Su Ippocrate, naturalmente, la letteratura è pressoché sconfinata. Si veda, a titolo d'esempio: J. Jouanna, Hippocrate, Paris, Librarie Arthème Fayard, 1992. Per 
in cui si tenta una sorta di correlazione anatomo-clinica ante litteram. Per esempio, nel trattato De locis in homine - già il titolo stesso è indicativo - l'ematuria è spiegata riferendosi a un danno nei 'vasi sanguigni' dei testicoli'. Inoltre, l'autore distingue sette tipi di infiammazioni diverse, ciascuna riferita alle 'vene' principali che originano dal cervello?. Il riferimento a Galeno, d'altra parte, è ancora più significativo e Morgagni lo fa, evidentemente, a ragion veduta. Da un lato, infatti, Galeno era stato certamente il principale protagonista dell'imposizione del modello umorale nella medicina occidentale ${ }^{10}$, ma, dall'altro, il suo approccio allo studio non solo della fisiologia, ma anche della patologia umana, implicava una profonda conoscenza dell'anatomia. Fra i suoi innumerevoli scritti, si possono citare le Anatomicae administrationes e il De locis affectis. Il primo è un trattato di anatomia descrittiva con l'esplicito intento, tuttavia, di essere utile alla «diagnosi dei mali» ${ }^{11}$. Il secondo riguarda, appunto, i 'luoghi' del corpo colpiti dalle malattie: si noti, per inciso, la corrispondenza fra «locis» del titolo galenico e «sedibus» del titolo morgagnano. Questo scritto, inoltre, seguiva, nella discussione delle malattie, il classico ordine a capite ad calcem, cioè dalla testa ai piedi, che sarà adottato anche da Morgagni nel suo De sedibus ${ }^{12}$. Galeno, infine, difendeva il ruolo dell'anatomia per la comprensione

quanto riguarda la scarsità delle conoscenze anatomiche in Ippocrate, si veda questa stessa opera, 308. In ogni caso, nel Corpus esiste un solo testo dal titolo Sull'anatomia, nel quale si descrive, in modo breve e piuttosto grossolano, l'anatomia del tronco umano: E.M. Craik, The Hippocratic treatise on anatomy, The Classical Quarterly 48 (1998), 135-167.

8 All'epoca non c'era distinzione, come ben noto, fra vene e arterie.

9 E. Littré, Oeuvres complètes d'Hippocrate. Traduction nouvelle avec le texte grec en regard, collationné sur les manuscrits et toutes les éditions; accompagné d'une introduction, de commentaires médicaux, de variantes et de notes philologiques; Suivie d'une table générale des matières, 10 voll., Paris, Ballière 1839-1861, vol. 6, 281-283, 273-274.

10 Anche se, è importante notare, lo fu solo indirettamente, cioè attraverso l'interpretazione e la cristallizzazione della sua opera avvenuta ad Alessandria d'Egitto nei primi secoli dell'era cristiana, portata a compimento dalla medicina araba e, in questa veste, importata in Europa nel corso del Medioevo: F. Zampieri, Il metodo anatomoclinico fra meccanicismo ed empirisimo, cit., 32-33.

11 I. Garofalo e M. Vegetti (a cura di), Opere scelte di Claudio Galeno, Torino, UTET, 1978, 141.

12 A. Cunningham, The Anatomist anatomis'd. An experimental discipline in Enlightenment Europe, Ashgate, Farnham, 2011, 188. 
delle malattie con le stesse parole che avrebbe usato Morgagni sin dal suo insediamento a Padova: «Solo attraverso la dissezione si può giungere a una corretta conoscenza dell'organizzazione e del funzionamento del corpo, in salute e in malattia» $»^{13}$.

La ricerca della «sede e delle cause», continua Morgagni, aveva poi ricevuto nuovo impulso in Italia a partire dalla rinascita dell'anatomia umana, cioè da quando si ristabilì l'uso di «aprire ... i corpi umani»» che si affermò sin dal Trecento presso la scuola bolognese per poi diffondersi in tutta la penisola e, successivamente, in tutt'Europa. Per quanto riguarda l'Italia, Morgagni cita Antonio Benivieni (1443-1502), medico fiorentino autore del De abditis nonnullis ac mirandis morborum et sanationum, pubblicato postumo nel 1507, che conteneva 111 osservazioni di carattere anatomo-patologico ${ }^{14}$, e Alessandro Benedetti (1450-1512), medico attivo fra Padova e Venezia, che fu fra i principali protagonisti dell'anatomia pre-vesaliana. Nel suo Anatomice, sive de historia corporis humani del 1502, Benedetti sosteneva che

Dall'anatomia deriva tutta la medicina, sia teorica che pratica, e la chirurgia. Se una malattia colpisce diverse parti o una in particolare nel corpo, se vi sono ascessi esterni o emorragie interne, se un paziente è ferito, o un suo arto è dislocato, nulla di tutto ciò può essere compreso senza l'anatomia ${ }^{15}$.

13 Citato in V. Nutton, Ancient medicine, $2^{\text {nd }}$ edn, London/New York, Routledge, 2013, 236. Ci riferiamo, qui sopra, alla celebre prolusione che Morgagni lesse il 17 marzo 1712 per il suo insediamento alla Cattedra di Medicina Teorica presso l'Università di Padova e che pubblicò nello stesso anno: G. B. Morgagni, Nova institutionum medicarum idea, Patavii, apud Josephum Coronam sub signo Coronae, 1712. Nel testo si trova, per esempio, un'affermazione ampiamente riportata nella letteratura storica riferita a Morgagni: «Affermeremo poi che non è possibile prospettare la natura e le cause di nessuna malattia senza le rispettive dissezioni dei cadaveri, né la cura senza le precauzioni e le specifiche prescrizioni»: L. Premuda (a cura di), Nova institutionum medicarum idea. Ristampa dell'edizione padovana del 1712 nel terzo centenario della nascita di G. B. Morgagni (1682-1771), Padova, La Garangola, 1982, 43.

14 A. Benivieni, De abditis nonnullis ac mirandis morborum et sanationum causis, Florentiae, Giunta, 1507. Morgagni citava il caso XXXVII del De abditis nella «lettera anatomo-medica» 39, par. 7, del De sedibus sui «Tumori paternaturali interni al ventre». Qui riportava che Benivieni osservò in un fanciullo un tumore fra le vene mesenteriche che le comprimeva fino a otturarle.

15 A. Benedetti, Historia corporis bumani sive anatomice, Venetiis, Bernardinus Guerralda Vercellensis, 1502, 3. 
Per quanto riguarda gli autori europei, Morgagni cita «Arveo», cioè William Harvey (1578-1657), medico inglese laureato a Padova nel 1602 che, nel 1628, proprio grazie alle sue ricerche anatomiche sull'uomo - oltre che, in realtà, a quelle anatomo-comparate - scoprì la circolazione del sangue ${ }^{16}$. Evidentemente, Morgagni sapeva che Harvey aveva intenzione di scrivere, settantenne, un volume intitolato Anatomia medica, composto delle esperienze anatomo-cliniche svolte lungo i suoi cinquant'anni di carriera, dal periodo di studi a Padova fino alla morte, volume che tuttavia non fu mai pubblicato ${ }^{17}$.

$\grave{E}$ proprio in questo periodo, cioè all'incirca fra la fine del Rinascimento e l'inizio dell'epoca moderna, che si diffonde l'uso della dicitura 'anatomia medica' e vengono pubblicate molte opere che si rifanno a quest'antica tradizione di correlazione anatomo-clinica. Per citare solo alcuni autori, basti pensare a Jean François Fernel (1497$1558)^{18}$, Johann Jacob Wepfer (1620-1695) ${ }^{19}$, Thomas Bartholin (161616802) ${ }^{20}$, Théophile Bonet (1620-1689) e Albrecht von Haller (1708-

16 W. Harvey, Exercitatio anatomica de motu cordis et sanguinis in animalibus, Francofurti, Sumptibus Gulielmi Fizteri, 1628.

17 G. Weber, Aspetti poco noti della storia dell'anatomia patologica tra '600 e '700, Firenze, Leo S. Olschki, 1997, 11-20.

18 Fernel scrisse i Pathologiae libri septem, nei quali si classificavano le malattie secondo gli organi colpiti e si sosteneva l'importanza di correlare i sintomi clinici con le ricerche autoptiche. Si veda J. M. Forrester, The Physiologia of Jean Fernel (1567), Philadelphia, American Philosophical Society, 1995.

19 Nelle Observationes anatomicae de apoplexia et exercitatio de eius loco affecto (1658), Wepfer fornì importanti osservazioni sulle malattie cerebrovascolari basate su storie cliniche e referti autoptici. Ad esempio, per primo mostrò che l'apoplessia era la conseguenza d'un aneurisma cerebrale; la sua Historiae apoplecticorum dello stesso anno è considerata un classico della ricerca sul tema; e nella Memoria Wepferiana (1727) descrisse le lesioni aterosclerotiche, fornendone una prima illustrazione. Si veda F. Zampieri, Il metodo anatomo-clinico fra meccanicismo ed empirismo, cit., 208.

20 Nel 1674, Bartholin pubblicò il De anatome practica ex cadaveribus morbosis adornanda consilium, che costituiva, più che un trattato di anatomia patologica vero e proprio (o meglio, di 'anatomia medica'), il progetto per la produzione di un'opera del genere. A proposito del quale scriveva: «Ho esposto le ragioni del mio progetto e $\mathrm{i}$ mezzi attraverso i quali sforzarsi di realizzarlo, in quanto composto dalle mie esperienze lungo molti anni e da quelle di altre persone provenienti da una Enciclopedia letteraria virtualmente universale»: T. Bartholin, De anatome practica ex cadaveribus morbosis adornanda consilium: cum operum autoris bactenus editorum catalogo, Hafniae, Sumptibus Petri Hauboldi, literis Georgii Godiani 1674, 39. 
$1777)^{21}$. Bonet pubblicò, nel 1679, il Sepulchretum ${ }^{22}$, che ebbe un notevole successo e che Morgagni, come ben noto, dichiara esplicitamente di aver preso a modello per il suo De sedibus.

Ora, il fatto che esistesse una consolidata tradizione, in tutto il corso della medicina occidentale, di studi 'anatomo-clinici', o perlomeno di tentativi, di varia natura, di comprensione delle basi anatomiche delle malattie, non significava che quest'approccio fosse stato esente da perplessità o critiche, anche feroci. In effetti, questi tentativi furono oggetto di una discussione metodologica cruciale che, come accennato qui sopra, ebbe inizio sin dal III secolo a.C., quando due celebri medici della cosiddetta Scuola Alessandrina, ovverosia Erofilo ed Erasistrato, stabilirono una netta distinzione fra una parte autenticamente 'scientifica' della medicina, basata sulla comprensione del funzionamento del corpo attraverso l'indagine autoptica, e una parte 'stocastica', connessa alla terapia e alla chirurgia, e che si basava, piuttosto, su quella che noi oggi definiremmo esperienza clinica ${ }^{23}$. Il primo tipo di medicina fu definito dogmatico, mentre il secondo empirico; questa spaccatura diede vita a delle vere e proprie sette, che si scontrarono in epoca ellenistica e romana e diedero vita a un nuovo, violento dibattito a partire dalla seconda metà del Seicento, con Marcello Malpighi (1628-1694), maestro 'spirituale' di Morgagni, fra i protagonisti.

\section{MEDICINA MECCANICISTA E MEDICINA EMPIRICA FRA 1650 E 1750}

In effetti, rispetto alla tradizione sia classica sia rinascimentale, nel corso del Seicento vi è l'introduzione di una nuova e, a suo modo, decisiva prospettiva nella concezione del corpo umano e delle sue malattie, cioè quella meccanicistica, di cui proprio Malpighi si fece fra i massimi

21 A. von Haller, Opuscola pathologica partim recusa partim inedita: quibus sectiones cadaverum morbosorum potissimum continentur. Accedunt experimenta de resouratione, quarta parte aucta, Lausannæ, Sumpt. Marci-Mich. Bousquet \& Soc., 1755.

22 T. Bonet, Sepulchretum sive anatomia practica, ex cadaveribus morbo denatis, proponens historias et observationes omnium bumani corporis affectuum, ipsorumque causas reconditas revelans, 2 voll., Genevae, sumptibus Leonardi Chouët, 1679.

$23 \mathrm{M}$. Vegetti, Tra il sapere e la pratica: la medicina ellenistica, in M. Grmek (a cura di), Storia del pensiero medico occidentale, 3 voll., Bari, Laterza 1993, vol. 1, 75-99. 
promotori in Italia. Tentando di semplificare e di fare ordine in un panorama altrimenti estremamente complesso e variegato - e alla cui complessità tenteremo, brevemente, di dar ragione nel corso di questo paragrafo - fra la seconda metà del Seicento e buona parte del Settecento si scontrarono due 'fazioni', per certi versi piuttosto ben delineate, per altri con più sfumata distinzione. Da una parte, si schieravano, se così si può dire, i medici 'meccanicisti' - variamente definiti come razionali o dogmatici in correlazione con l'antica disputa ellenistica, ma anche 'neoteorici', in quanto sostenitori di teorie e metodi nuovi, oppure iatromeccanici, iatromatematici, iatrofisici e iatrochimici, a seconda dell'enfasi posta su di un particolare aspetto metodologico o concettuale - mentre dall'altra si schieravano i medici 'empirici'. I primi erano sostenitori della ricerca anatomica per la comprensione delle 'macchine' del corpo, funzionale, a sua volta, per la comprensione e cura delle malattie. I secondi, invece, avversavano l'anatomia, perlomeno come mezzo per migliorare la terapia, e si richiamavano alla necessità di un esclusivo, costante e continuo studio delle malattie al letto del malato, basato, quindi, sull'osservazione e catalogazione dei sintomi, del decorso clinico e degli effetti delle terapie farmacologiche. Il caposcuola della iatromeccanica, perlomeno in Italia, ma per certi versi anche a livello europeo, fu Marcello Malpighi. Il massimo rappresentante della medicina empirica, invece, fu senz'altro l'inglese Thomas Sydenham (1624-1689).

I primi si ispiravano a Galileo Galilei (1564-1642) - in particolare ai suoi assunti fondamentali secondo i quali la natura fosse composta di 'materia' e 'movimento' e potesse essere studiata e compresa con la matematica e la geometria - e al filosofo francese René Descartes (1596-1650), meglio noto come Cartesio, in quanto padre della concezione meccanicistica moderna e, in particolare, di quella secondo la quale il corpo umano fosse analogo a una macchina. I secondi, cioè i medici empirici, si ispiravano principalmente al filosofo, nonché medico egli stesso, John Locke (1632-1704), padre, appunto, dell'empirismo moderno - e in particolare alla concezione secondo la quale la conoscenza umana fosse strutturalmente incapace di indagare le cause remote o interne dei fenomeni, ma dovesse quindi restare limitata alla descrizione dei fenomeni per come si manifestavano all'osservazione. Tradotto in campo medico, questa filosofia significava, necessariamente, limitarsi all'osservazione clinica.

Tuttavia, come vedremo brevemente, entrambi gli schieramenti si richiamavano al primato dell'osservazione nella ricerca scientifica, sebbene le dessero un ruolo diverso, mentre nei confronti della tradi- 
zione, o meglio della medicina degli antichi, l'atteggiamento delle due fazioni era in certi casi opposto, in altri sovrapponibile. Entrambi questi elementi rendono ragione del fatto che siamo di fronte, come già detto, a fazioni diverse, ma dai contorni sfumati.

Per quanto riguarda il primato della tradizione, si può sostenere che molti, sia fra i meccanicisti che fra gli empiristi, si richiamavano alla filosofia di Francesco Bacone (1561-1626): Locke e Sydenham da una parte, lo stesso Malpighi e il chimico e fisico meccanicista Robert Boyle (1627-1691) dall'altra, lo consideravano come un fondamentale precursore della rivoluzione scientifica. I primi, per il suo richiamo all'osservazione come base della conoscenza, i secondi per la sua epistemologia fondata sia su osservazione che su sperimentazione ${ }^{24}$. In Bacone, il «metodo storico» si differenziava fondamentalmente dal «metodo filosofico» ed era ad esso superiore: il primo si basava sull'osservazione dei fenomeni naturali nel loro svolgersi temporale; il secondo, invece, su categorie concettuali non sempre riconducibili all'esperienza. Sydenham, sostenendo che delle malattie si dovesse osservare esclusivamente la 'storia naturale', si rifaceva proprio alla metodologia storica di Bacone $^{25}$. Boyle e i meccanicisti, invece, consideravano il metodo storico come punto di partenza dell'analisi sperimentale, cioè come fonte di dati empirici sui quali, successivamente, costruire modelli, teorie, ipotesi ed esperimenti - ciò che era previsto dalla stessa metodologia baconiana. Per esempio, secondo Malpighi,

Lo stesso possiamo dire delle macchine del nostro corpo, che sono alla base della medicina: atteso che queste sono composte di corde, di filamenti, di travi, di leve, di tele, di fluidi scorrenti, di cisterne, di canali, di feltri, di crivelli, e di somiglianti macchine. L'uomo, esaminando queste parti con l'anatomia, con la filosofia e con la meccanica, e procedendo anche a priori, è arrivato a formulare modelli, con li quali pone sotto l'occhio la causalità di quell'effetto, e ne rende la ragione a priori, e con la serie di queste, aiutato dal discorso, intendendo il modo dell'operare della natura, fonda la fisiologia e la patologia, e successivamente l'arte della medicina ${ }^{26}$.

24 D.E. Wolfe, Sydenham and Locke on the limits of anatomy, Bulletin of the History of Medicine 35 (1961), 193-220, qui 194.

25 F. Duchesneau, Les modéles du vivant de Descartes à Leibniz, Paris, Librarie Philosophique J. Vrin, 1998, 243.

26 M. Malpighi, Risposta del Dottor Marcello Malpighi alla lettera intitolata De 
Parole quasi identiche furono utilizzate da un altro grande rappresentante della iatromeccanica della generazione successiva a quella di Malpighi, l'olandese Hermann Boerhaave (1668-1738), che nelle Istitutiones medicae del 1708 scriveva: «Si trovano in effetti nei corpi sostegni, colonne, fasci, bastioni, tele, corde, leve, pulegge, presse, setacci, filtri, canali, abbeveratoi, serbatoi ${ }^{27}$. Questa concezione rifletteva un'idea espressa da Bacone nel suo Advancement of learning, nel quale divideva la 'storia naturale' e lo studio della natura in tre parti. Primo, nella sua costanza. Secondo, nelle sue eccezioni e variazioni, come ad esempio la teratologia, interessata a vitelli ed esseri uomini nati con due teste, etc. Terzo e ultimo, in quanto alterata o modificata dall'uomo mediante macchine e congegni costruiti per raggiungere determinati obiettivi, cosicché lo studio sperimentale della natura era definito anche, significativamente, bistoria mechanica ${ }^{28}$.

Tuttavia, poteva esistere una certa differenza anche fra lo sperimentalismo di Boyle e quello dei meccanicisti. Boyle, pur usando modelli meccanici - si pensi alla sua macchina pneumatica per studiare il rapporto fra combustione e respirazione - rimaneva fondamentalmente uno scettico ${ }^{29}$. Egli, cioè, sosteneva che le teorie scientifiche costruite grazie alla sperimentazione non fossero mai definitive, perché necessitavano d'una continua verifica attraverso nuovi esperimenti e potevano, attraverso questi, essere continuamente implementate o confutate. I medici meccanicisti, dal canto loro - o perlomeno soprattutto quelli di prima generazione, se così possiamo dire, cioè operanti nell'ultima parte del Seicento - dimostravano, spesso, una fiducia maggiore negli esiti di tali sperimentazioni, sostenendo che potessero portare a scovare, una volta per tutte, i più nascosti segreti della natura. Tuttavia, altri fecero ben attenzione a non cadere nella trappola della concezione della medicina come di una scienza esatta, sottolineandone a più riprese

recentiorum medicorum studio dissertatio epistolaris ad amicum, in M. Malpighi, Marcelli Malpighi opera posthuma, 2 voll., Londini, Impensis A. \& J. Churchill, ad Insigne Nigri Cygni in Vico dicto Pater-noster-Row, 1697, vol. 2, 110.

$27 \mathrm{H}$. Boerhaave, Institutiones medicae in usu annuae exercitationes domestic digestae, Lugduni Batavorum, apud J. Van Linden, 1708, 40.

28 Cfr. D. Garber, La rivoluzione scientifica. Introduzione, in AAVV, Storia della scienza, 10 voll., Roma, Treccani, vol. 4, 2002, 731-749, qui 735.

29 C. Pighetti, L'influsso scientifico di Robert Boyle nel tardo '600 italiano, Milano, FrancoAngeli, 1988, 30-31. 
il sostanziale carattere congetturale. Fra i tanti, un esempio significativo può essere rappresentato dal medico campano Leonardo Di Capua (1617-1695). In un'opera nella quale tentava di dimostrare, punto per punto, la superiorità della medicina neoterica su quella antica, egli sottolineava, allo stesso tempo, i limiti costitutivi dell'intelletto umano che rendevano incerti anche i risultati dell'approccio neoterico. Per far fronte a tali limiti, tuttavia, sosteneva che l'unica possibilità, per i medici, fosse quella di fondarsi sul metodo galileiano ed essere esperti di 'geometria' come Cartesio ${ }^{30}$. Boerhaave, invece, andò progressivamente aderendo, in particolare sotto l'influsso dell'opera di Isaac Newton (1642-1726), a una sorta di meccanicismo critico, secondo il quale tale approccio aveva un valore fondamentalmente euristico ${ }^{31}$. La teoria della gravitazione universale, infatti, poggiava su una sorta di agnosticismo metodologico. Si trattava, cioè, d'una legge fisica generale derivata per induzione da osservazioni empiriche, legge nella quale vi era, allo stesso tempo, una sospensione di giudizio sulla natura dell'agente causale in virtù del quale i fenomeni manifestavano regolarmente quel comportamento. Sostenere che l'attrazione di due corpi nell'universo fosse proporzionale al prodotto delle loro masse e inversamente proporzionale al quadrato della loro distanza non implicava, infatti, una qualche ipotesi a riguardo della natura della gravitazione e dell'attrazione. Il calcolo degli effetti e l'inferenza matematica che si potevano ricavare dai fenomeni erano sufficienti a stabilire un sistema di leggi senza il supporto ontologico della natura stessa degli agenti causali, sconosciuta e metodologicamente ipotizzata come non conoscibile ${ }^{32}$. Boerhaave applicava questo stesso agnosticismo metodologico alla natura dei corpuscoli del corpo. Proprietà come massa, portata, impenetrabilità, inerzia, indivisibilità fornivano le condizioni sufficienti per la concezione geometrica degli effetti meccanici osservabili, ma rimanevano, in sé stesse, inconoscibili. Per questo, Boerhaave criticava gli iatromeccanici seguaci di

30 L. Di Capua, Parere del signor Lionardo Di Capua divisato in otto ragionamenti, ne' quali partitamente narrandosi l'origine, e'l progresso della medicina, chiaramente l'incertezza della medesima si fa manifesta, Napoli, Per Giacomo Raillard, 1681, 72. Vedi anche S. Scarabella, Di Capua, Leonardo, in Dizionario Biografico degli Italiani, Roma, Istituto della Enciclopedia Italiana, 1991, vol. 39, 714.

31 F. Zampieri, Il metodo anatomo-clinico fra meccanicismo ed empirismo, cit., 62.

32 F. Duchesneau, La physiologie des lumiéres. Empirisme, modèles et théories, The Hague/Boston/New York, Martinus Nijhoff Publishers, 1982, 108. 
Cartesio per aver tentato un'applicazione a priori del metodo meccanista in medicina. La medicina, al contrario, doveva ricorrere ai modelli meccanici solo seguendo un percorso a posteriori3 ${ }^{33}$. L'approccio newtoniano di Boerhaave affiorava in modo particolarmente chiaro in una sua orazione accademica del 1715, De comparando certo in physicis, all'inizio della quale si esprimeva come segue:

Tenterò di difendere l'idea che i princìpi delle cose ci sono del tutto nascosti, che noi possiamo cogliere le proprietà dei corpi solamente attraverso le caratteristiche osservabili che conosciamo con l'esperienza o quelle che possiamo conoscere partendo dalle caratteristiche esplorate seguendo la via stabilita dalla solidità del ragionamento geometrico ${ }^{34}$.

Parole, queste, che non sarebbero dispiaciute a molti dei medici empirici del tempo, oltre che a quelli dichiaratamente iatromeccanici.

Fra medici iatromeccanici e medici empirici, quindi, potevano anche esserci punti di contatto profondi. Ritengo sia estremamente significativo, da questo punto di vista, che Sydenham, Locke e Boyle si menzionassero a vicenda, come se fra i primi due e il terzo non esistessero concezioni irrimediabilmente inconciliabili. Sydenham dedicava il suo Methodus curandis febres ${ }^{35}$ a Boyle. Boyle dedicava le sue Memoirs for the natural history of bumane blood al «very ingenious and learned Dr. J. L.», cioè, appunto, a Locke ${ }^{36}$. Locke, infine, nella Epistle to the reader del suo celeberrimo An essay on buman understanding si riferiva a Sydenham e Boyle come ai suoi «master-builders» $»^{37}$. Non si trattava, quindi, solo di scontri fra dottrine opposte, perché si assisteva anche a discendenze comuni, contaminazioni reciproche, scambi di ruoli. Altrettanto significativo, sempre in relazione a questa possibile inter-

33 F. Duchesneau, La physiologie des lumiéres, cit., 112.

34 H. Boerhaave, Sermo academicus de comparando certo in physicis, quem babuit in Academia Lugduno-Batava, quuum octavo februarii, anno 1715 rectoratum Academiae deponeret, Lugduni Batavorum, apud P. Van der Aa, 1715, 2-3.

35 T. Sydenham, Methodus curandis febres propriis observationibus superstructa, Londini, Impensis J. Crook, apud quem veneunt sub Signo Navis in Coemeterio D. Pauli, 1666.

36 R. Boyle, Memoirs for the natural history of bumane blood, in T. Birch (ed.), The works of the honorable Robert Boyle, 5 vols, London, Millar, 1744, vol. 4, 160-189. set, 1690 .

37 J. Locke, An Essay on Human Understanding, London, Printed for the Bas- 
cambiabilità, che Leonardo Di Capua ammettesse la possibilità che i sostenitori della medicina razionale, proprio sulla base della superiorità dell'osservazione e dell'esperienza rispetto ai modelli teorici, si convertissero alla scuola empirica:

Ma per dar cominciamento alla Empirica, ella a prima vista sopra una saldissima base aver sembra le sue fondamenta, che è la sperienza; non solamente per la bassa gente, ma per gl'istessi medici razionali cotanto stimata, e a capital tenuta; che una delle due colonne della medicina chiamar la sogliono; essendo l'altra, secondo lor sentimenti, la ragione. Anzi huomini chiarissimi della setta de' Razionali cotanto agli Empirici nemica ... abbandonando la lor setta Razionale, e lasciate affatto le ragioni, alla sola sperienza degli Empirici ricoverati alla fine si rifuggono; ed altri, comechè perseverino nella setta de' Razionali, pur manifestamente confessano esser soventi volte da antiporre la sperienza alla ragione, e dicono, che ove d'una parte della ragione, e d'altra la sperienza il contrario ne persuadono, che allora il medico lasciar debba affatto la ragione, e la sperienza solamente seguire ${ }^{38}$.

Un ulteriore esempio significativo è rappresentato da John Barker (1708-1748), medico del Westminster Hospital (1746-1747), poi ingaggiato nelle forze armate britanniche nei Paesi Bassi (1747-1748), che pubblicò, poco prima della morte, An essay on the agreement between ancient and modern physicians. In tale lavoro Barker tentava di superare il contrasto fra meccanicisti ed empiristi proprio dimostrando la corrispondenza fra Ippocrate e medicina empirica, rappresentata da Sydenham da una parte, e fra Galeno e medicina neoterica, rappresentata da Boerhaave dall'altra. Il sottotitolo, infatti, era Comparison between the practice of Hippocrates, Galen, Sydenham, and Boerbaave in acute diseases. Qui tentava di dimostrare che, in particolare nelle malattie acute, esistesse una sola regola di cura e questa regola, stabilita da Ippocrate per primo, fu seguita da tutti i migliori medici successivi. Non c'era quindi alcun contrasto fra scuole diverse, perché il trattamento migliore delle malattie era uno e uno soltanto. In questo caso, a differenza dell'esempio portato da Di Capua, si trattava di conciliazione fra meccanicisti ed empiristi, piuttosto che di conversione dei primi nei secondi, ma il fattore cruciale in gioco era, pressappoco, lo stesso. Di

38 L. Di Capua, Parere del signor Lionardo Di Capua divisato in otto ragionamenti, cit., 102. 
Capua, cioè, parlava di 'esperienza', mentre Barker di 'trattamento' delle malattie: in entrambi i casi si trattava, quindi, dell' aspetto pratico che permetteva di gettare un ponte tra le due diverse fazioni. E a proposito di «pratica», si può citare, infine, anche Giorgio Baglivi (16681707), uno dei più autorevoli sostenitori delle dottrine iatromeccaniche in Italia e allievo dello stesso Malpighi a Bologna. Ebbene, secondo Baglivi l'osservazione e l'esperienza, nella terapeutica, avevano un ruolo ben più importante dei modelli meccanici, perché le malattie erano determinate più da un disturbo nei fluidi del corpo, che da una lesione dell'organo comparabile a un danno meccanico ${ }^{39}$. Nel suo De praxi medica del 1696, inoltre, basandosi sull'idea d'una sostanziale indipendenza della clinica rispetto alle ricerche biologiche, Baglivi difendeva un approccio terapeutico fondato essenzialmente sul concetto ippocratico di vix medicatrix naturae, secondo il quale il corpo avesse in sé la forza e la capacità di curarsi ${ }^{40}$. Si badi bene che riferirsi a Ippocrate e alla scuola ippocratica spesso costituiva una sorta di esplicito o implico schieramento nei confronti della medicina empirica. La scuola ippocratica, infatti, era vista come il massimo esempio di medicina clinica, cioè rivolta principalmente all'osservazione - e alla cura - delle malattie al letto del malato.

Per quanto riguarda l'atteggiamento dei due schieramenti nei confronti della tradizione, le dinamiche in gioco possono essere schematizzate come segue. Il primato dell'osservazione costituiva un elemento comune per cui, in linea di principio, sia i meccanicisti sia gli empiristi rifiutavano la tradizione, o meglio rigettavano il principio di autorità che, soprattutto in ambito accademico, ad essa si legava ${ }^{41}$. Il già citato Di Capua, per esempio, si esprimeva come segue:

39 M. D. Grmek, La notion de fibre vivante chez les médecins de l'école iatrophysique, Clio Medica 5 (1970), 297-318, qui 310.

40 G. Baglivi, De praxi medica ad priscam observandi rationem revocanda. Libri duo. Accedunt dissertationes novae, Romae, Typis Dominici Antonii Herculis, 1696.

41 L'epoca moderna, come ben noto, è stata caratterizzata dalla nascita delle accademie scientifiche - basti pensare alla celeberrima Accademia del Cimento di Firenze, ma anche a quella dei Lincei di Roma o degli Inquieti di Bologna, alla Royal Society di Londra, all'Académie royale des sciences di Parigi e così via - che si costituirono come centri di propulsione di nuove idee. Le università, dal canto loro, restavano spesso ancorate a programmi di insegnamento tradizionali - risalenti perlopiù al Medioevo - che richiesero molto più tempo per essere rinnovati. Si vedano, ad esempio, M. Cavazza, 
Ma perché dobbiamo mai sempre noi con folle ostinazione lasciarci trarre al reverendissimo parere degli antichi? Forse non son state lor molte cose a grado, ch'a noi spiacevoli ora sono, ed affatto noiose? ... $\mathrm{O}$ forse sarà egli colpa dei moderni l'esser'eglino nati dopo gli antichi? Ma se ciò è fallo, e colpa, certamente commiserla in prima coloro, $\mathrm{i}$ quali da' sentimenti de' loro più antichi maestri tralignando ... osarono in prima novelli scolari ribellarsi a loro antichi maestri, e darne nocevole esempio di temerario ardimento. Imperciocchè ogni antico a' tempi suoi fu moderno .... Saran dunque da vituperare Platone, Antistene, Eschine, i quali posto in non cale le vecchie scuole, che allora nella Grecia fiorivano, a quella di Socrate, che nuova era, per imprender filosofia coraggiosamente s'en girono? ${ }^{42}$

Molto bella, trovo, l'espressione qui citata: «ogni antico a' tempi suoi fu moderno». Tuttavia, i meccanicisti si potevano ispirare agli antichi perché vi erano stati innumerevoli esempi, nella medicina classica greco-romana, di tentativi analoghi a quelli in cui si cimentavano loro stessi, cioè ricerche anatomiche, come già detto, ma perfino concettualizzazioni meccaniche e idrauliche del corpo, o misurazioni di parametri fisiologici, come la frequenza cardiaca ${ }^{43}$. Confortati da questi precedenti storici, inoltre, i medici neoterici potevano utilizzare fonti antiche per dimostrare, negli ambienti accademici più conservatori, che loro stessi non stavano facendo nulla di così drammaticamente nuovo rispetto, appunto, ai venerandi autori del passato. In questo caso, si trattava, perlopiù, di espedienti retorici per far fronte ad ambienti più o meno ostili al rinnovamento. I medici empirici, per canto loro, basandosi principalmente sull'osservazione clinica, non potevano disdegnare, per così dire, la straordinaria tradizione ippocratica, ma anche certa tradizione araba,

Settecento inquieto. Alle origini dell'Istituto delle Scienze di Bologna, Bologna, Il Mulino, 1990, e M. P. Donato e J. Kraye (a cura di), Conflicting duties: Science, medicine and religion in Rome, 1550-1750, London/Torino, The Warburg Institute/Nino Aragno Editore, 2009.

42 L. Di Capua, Parere del signor Lionardo Di Capua divisato in otto ragionamenti, cit., 36-37.

43 Si pensi, in particolare, proprio ai medici alessandrini Erofilo ed Erasistrato. Erofilo, per esempio, aveva elaborato un'intricata teoria dei 'polsi' e si serviva, a quanto pare, di una sorta di orologio ad acqua per misurare i diversi tipi di frequenza cardiaca, sia in salute e a seconda delle diverse fasce d'età, sia in malattia: H. Von Staden, Herophilus. The art of medicine in early Alexandria, Cambridge/New York, Cambridge University Press, 1989, 281-285. 
basata, appunto, su dettagliate e puntuali descrizione di sintomi, sindromi e decorsi clinici. La medicina, infatti, oltre a essere scienza in senso stretto (quella che oggi chiameremmo ricerca di base), è anche, forse soprattutto, esperienza viva sul paziente. E l'esperienza e l'osservazione dei sintomi clinici costituiscono una pratica che ha una sorta di validità universale, cosicché la corretta descrizione di un sintomo clinico, che sia stata fatta da Ippocrate o da un 'moderno', formalmente poteva (e può) avere lo stesso valore. Inoltre - e anche questo è un punto cruciale che, in un certo modo, ha caratterizzato tutta la storia della medicina, compresa quella contemporanea - il fine di questa scienza è, essenzialmente, la cura: se un farmaco, quindi, dà prova della sua efficacia, risulta di secondaria importanza se provenga da un sapere antico o moderno, o sapere come ciò avvenga attraverso la fisiologia, la chimica o l'anatomia. L'importante, in sostanza, è che funzioni, cioè che guarisca il paziente. Quindi, come giustamente è già stato fatto notare, «... assai più complessa è la storia dell'‘abbandono' di Galeno, perché la pratica medica accumulava esperienze, antiche e moderne, e, pur correggendo le osservazioni del passato, non poteva rifiutarne gli aspetti ancora validi, l'ammaestramento tradizionale, lo spirito empirico» ${ }^{44}$. Basti pensare, solo a titolo d'esempio, che ancora nel 1766 si ristampava a Londra un antico trattato del medico arabo noto in Occidente come Rhazes (865-925), sul vaiolo e il morbillo perché queste due malattie vi erano descritte e differenziate correttamente da un punto di vista clini$\mathrm{Co}^{45}$. Si era in un periodo, tra l'altro, in cui il vaiolo era particolarmente virulento e una sua corretta descrizione, perciò, poteva ancora risultare utile a prescindere dalla sua antichità. Rispetto a questa natura universale della medicina, quindi, gli empirici si trovavano a fare i conti con la tradizione in modo più agevole rispetto ai medici neoterici. Tuttavia, come già detto, ciò non impedì a molti di loro di rigettare la tradizione per sostituire le vecchie con nuove osservazioni, considerate più dettagliate e veritiere, perlomeno perché basate esclusivamente sulla ripetuta esperienza diretta dell'osservatore.

44 C. Pighetti, Un dialogo di Domenico Guglielmini restituito alla critica da Giambattista Morgagni, in V. Cappelletti e F. Di Trocchio (a cura di), De sedibus, et causis. Morgagni nel centenario, cit., 125-133, qui 125.

45 I. Channing (a cura di), Razhes, De variolis et morbillis, arabice et latinae; cum aliis nonnullii eiusdem argumenti, Londini, Excudebat Guilielmus Bowyer, 1766. 
Giungiamo, ora, a fornire qualche dettaglio in più sui protagonisti principali di questo complesso scenario. Come già detto, fra gli iatromeccanici merita senz'altro un ruolo di primo piano Marcello Malpighi. Docente di medicina a Bologna, Pisa, Messina e, infine, archiatra di papa Innocenzo XII (1615-1700) a Roma, fra gli anni Sessanta e Novanta del Seicento $« . .$. diede contributi fondamentali all'anatomia virtualmente in ogni settore del mondo animale e vegetale ${ }^{46}$. In sostanza, secondo Malpighi le parti solide del corpo umano erano composte di strutture meccaniche che obbedivano alle leggi della geometria e della matematica. Quelle fluide, allo stesso modo, erano composte di particelle microscopiche il cui comportamento era determinato dalla loro forma geometrica. Per la comprensione del corpo umano, dunque, era necessaria la cosiddetta 'anatomia sottile', cioè una ricerca anatomica che, attraverso l'uso del microscopio, potesse osservare i costituenti più minuti della macchina organica. Per la comprensione delle funzioni fisiologiche, inoltre, ci si poteva rivolgere anche a modelli meccanici che riproducessero le caratteristiche di alcuni organi o parti del corpo, in modo da poter simulare sia i processi normali che i disordini riconducibili a una qualche rottura o disfunzione. Oltre ai capillari sanguigni e ai glomeruli renali, scoperte per le quali Malpighi è universalmente noto, il medico bolognese scoprì l'esistenza di una miriade di strutture ghiandolari nei diversi organi e tessuti che lo portarono a riformare, dall'interno, la teoria umorale e a proporre un nuovo modello del corpo come, appunto, «macchina ghiandolare $»^{47}$. Il sangue, circolando nel corpo, veniva filtrato dalle diverse ghiandole producendo i diversi umori, che non erano più quattro come nello schema classico. Operando come filtri meccanici, le ghiandole salivari producevano la saliva, il pancreas il succo pancreatico, i testicoli lo sperma, il fegato la bile, il cervello il «sugo nervoso», i reni l'urina e così via ${ }^{48}$. Ciascun tipo di particella, caratterizzata da una forma geometrica speci-

46 D. Bertoloni Meli, The new anatomy of Marcello Malpighi, in D. Bertoloni Meli (a cura di), Marcello Malpighi anatomist and physician, Firenze, Leo S. Olschki, 1997, 21-62, qui 21.

47 D. Bertoloni Meli, The new anatomy of Marcello Malpighi, cit.

48 D. Bertoloni Meli, The new anatomy of Marcello Malpighi, cit., 44-45. Fedele a un approccio puramente meccanicistico, Malpighi negò la possibilità che la secrezione ghiandolare avvenisse anche con l'aiuto di un qualche processo chimico come la fermentazione: D. Bertoloni Meli, Mechanism, experiment, disease. Marcello Malpighi and seventeenth-century anatomy, Baltimore, John Hopkins University Press, 2012, 81. 
fica, veniva trattenuta da una specifica specie ghiandolare attraverso la forma corrispondente del proprio filtro costitutivo ${ }^{49}$.

Malpighi fu il caposcuola di una generazione di medici che Italia diffusero ampiamente questo nuovo approccio. Fra i suoi discepoli prediletti, possiamo citare Antonio Maria Valsalva (1666-1723), che svolse ricerche fondamentali sull'orecchio umano, concepito come una sorta di macchina sonora ${ }^{50}$; Giovanni Alfonso Borelli che trattò in modo geometrico il movimento interno ed esterno degli animali; Lorenzo Bellini $(1643-1704)^{51}$; il già citato Baglivi; Domenico Guglielmini (1655-1710) che passò dall'idraulica alla medicina, applicando la teoria particellare dei fluidi allo studio del sangue ${ }^{52}$; e, infine, Antonio Vallisneri (1661-1730), che svolse studi importanti non solo di medicina, ma anche di scienze naturali ${ }^{53}$. Si noti che Valsalva fu il maestro di Morgagni, al quale trasmise l'approccio anatomico di Malpighi basato sull'anatomia sottile, mentre Guglielmini e Vallisneri furono suoi amici ed entrambi docenti a Padova, dove, come Morgagni, furono direttamente coinvolti nella disputa fra iatromeccanica e medicina empirica ${ }^{54}$.

49 Fu la filosofia di Cartesio che probabilmente influì in modo decisivo per la focalizzazione di Malpighi sulle ghiandole. Ne L'bomme del 1667, Cartesio elaborava esplicitamente il modello ghiandolare come filtro fondamentale per separare i costituenti del sangue, modello che fu poi ripreso letteralmente da Giovanni Alfonso Borelli (1608-1679), un altro fondamentale rappresentante del meccanicismo medico italiano, nel suo De motu animalium, Roma, A. Bernabo, 1680-1681. Si veda F. Zampieri, Il metodo anatomo-clinico fra meccanicismo ed empirismo, cit., 84-85.

50 A.M. Valsalva, De aure bumana tractatus, in quo integra ejusdem auris fabrica, multis novis intentis, E cononismis illustrata, describitur; omnique eius partium usus indanatur. Quibus interposita est musculorum uvuale, atque pharyngis nova descriptio, et delineatio, Bononiae, Typis Costantini Pisarii ad S. Michelem propre Archigymnasium, 1704.

51 Antonio Cocchi (1695-1758), docente di anatomia nello Studio fiorentino, nella lunga prefazione ai Discorsi di anatomia di Bellini, lo definisce come il padre della iatromeccanica: «... non si può però negare che la lode d'avere il primo formato un sistema di medicina mecanica, atto principalmente a spiegare i fenomeni delle malattie e a ben dirigere il medico nella scelta delle sue operazioni, sia stata felicemente occupata dal nostro Bellini»: A. Cocchi, Prefazione, in A. Bellini, Discorsi di anatomia, Firenze, Stamperia di Francesco Moücke, 1741, VII-XXXI, qui VIII.

52 D. Guglielmini, De sanguinis natura E constitutione exercitatio physicomedica, Venetiis, ex typographia Andreae Poleti, 1701.

53 F. Zampieri, Il metodo anatomo-clinico fra meccanicismo ed empirismo, cit., 353-355.

54 F. Zampieri, Il metodo anatomo-clinico fra meccanicismo ed empirismo, cit., 353-355, 366-375. 
In Europa, il già citato Boerhaave fu uno dei più illustri rappresentanti dell'approccio iatromeccanico. Concepiva il corpo come una macchina le parti solide del quale erano costituite da vasi costruiti per contenere, dirigere, modificare, separare e secernere i fluidi (composti, a loro volta, di particelle). Tutte le altre parti solide costituivano le strutture di supporto a queste funzioni fondamentali. Con ogni probabilità, come lo stesso Malpighi, si era ispirato al meccanicismo cartesiano. Per esempio, riecheggiava il cogito ergo sum di Cartesio nelle sue lezioni di psicologia sostenendo che: $I d$, quod cogitat, mens dici$t_{u r^{5}}$. Nel 1703, Boerhaave teneva una lettura inaugurale dal titolo quanto mai significativo, De usu ratiocinii mechanici in medicina, che costituiva una professione di fede nei confronti della iatromeccanica. La quale iniziava con questa frase inequivocabile: «I meccanicisti sono coloro che, attraverso calcoli matematici basati su osservazioni o premesse razionali, spiegano le operazioni dei corpi con le loro masse, configurazioni e velocità ${ }^{56}$.

In Germania, Friedrich Hoffmann (1660-1742) concepiva il corpo come un sistema idraulico, funzionante attraverso etere o fluido nervoso. Tale spirito era meccanicamente concepito come un «gas flammeo generato dalla fermentazione del sangue» che raggiungeva il cervello, creando così lo spirito animale, a sua volta primum movens di sensazioni, movimenti e pensieri dell'uomo ${ }^{57}$. Tale spirito era di «natura astrale» perché composto delle stesse particelle finissime dell'etere, del quale erano costituite anche le stelle del cielo, e per questa analogia il corpo umano era in costante collegamento con gli astri e veniva astrologicamente influenzato da essi ${ }^{58}$. Come fa notare Francesco Paolo De Ceglia, non è sorprendente constatare la presenza di concezioni astrologiche in teorie di natura meccanicistica: «L'astrologia affiancò la medicina, tanto più in quanto vivificata dagli strumenti fornitile dalla

55 G. A. Lindeboom (a cura di), Boerhaave and his time. Papers read at the international symposium in commemoration of the trecentenary of Boerhaave's birth, Leiden, 15-16 November 1968, Leiden, E. J. Brill, 1970, 93.

$56 \mathrm{H}$. Boerhaave, De usu ratiocii mechanici in medicina, oratio babita in auditorio magno 24 septembris a. 1702, cum tertii suae stationis anni labores auspicaretur, Lugduni Batavorum, apud H. Teering, 1703, 2.

57 F. P. De Ceglia, I fari di Halle. George Ernst Stahl, Friedrich Hoffmann e la medicina europea del primo Settecento, Bologna, Il Mulino, 2009, 276.

58 F. P. De Ceglia, I fari di Halle, cit., 277-278. 
scienza newtoniana, che, se non altro per giustificare la forza gravitazionale, ebbe a parlare di 'occulti' influssi esercitati a distanza» ${ }^{59}$.

In Inghilterra, per citare solo alcuni nomi, noti iatromeccanici furono Archibald Pitcairne (1652-1713) e Richard Mead (1673-1754).

Se Malpighi fu il più rappresentativo e significativo dei medici neoterici, il clinico inglese Thomas Sydenham, come già detto, fu il più importante rappresentante della medicina empirica. Egli fece proprio quest'approccio rifiutando sia la pretesa di indagare i fenomeni fisiologici e patologici attraverso l'anatomia, in quanto costituiva un tentativo di indagarne le cause interne, sia quella di utilizzare modelli meccanici, in quanto si trattava di un'astrazione illegittima per l'intelletto umano. In questo modo, Sydenham si poneva in esplicito e netto contrasto con la medicina neoterica. Se Malpighi sviluppò l'anatomia in tutte le sue potenzialità sperimentali, Sydenham fu un clinico straordinario, cioè un finissimo osservatore dei fenomeni che le malattie manifestavano all'esterno del paziente, come febbre, tosse, dolore, e così via, e un altrettanto abile terapeuta. Non a caso, fu nominato, in vita, l'«Ippocrate britannico». La sua medicina si sviluppava nella descrizione dettagliata del decorso clinico delle malattie, degli effetti dei farmaci, della storia dei pazienti prima, durante e dopo le malattie, offrendo vere e proprie narrazioni realistiche. Queste osservazioni, poi, erano ordinate all'interno di categorie nosologiche che tentavano di non concedere nulla a ipotesi non ricavabili da ciò che si era potuto osservare. In breve, secondo Sydenham «... noi sappiamo ciò che ci insegnano i nostri cinque sensi .... La filosofia della medicina consiste nell'applicarsi alle storie naturali delle malattie e nell'uso dei rimedi che possono scacciarle; e $l^{\prime} E s p e r i e n z a$ è la sola guida $\aleph^{60}$. Un famoso aneddoto è molto significativo a proposito della considerazione che l'Ippocrate britannico aveva dell'anatomia. Si narra infatti che Hans Sloane (1660-1753), medico e naturalista inglese, andasse a studiare presso Sydenham portando una lettera di presentazione che lo definiva come «studioso maturo, buon botanico e abile anatomista». Vista la lettera, Sydenham lo rimbeccò:

Questo è molto bene, ma io non voglio fare Anatomia-Botanica, assurdità! Sir, conosco una vecchia di Coven Garden che conosce la

59 F. P. De Ceglia, I fari di Halle, cit., 279.

60 Citato in D. E. Wolfe, Sydenham and Locke on the limits of anatomy, cit., 196. 
botanica molto meglio di lei, e per quanto riguarda l'Anatomia, anche il mio macellaio può sezionare perfettamente un'articolazione; no, giovanotto, queste sono cose inutili: lei deve andare al letto del malato, solo lì può imparare cosa sia la malattia ${ }^{61}$.

Lo studio della natura a occhio nudo, senza strumenti, era una sorta di «imperativo morale» per Sydenham ${ }^{62}$. A riguardo, v'è uno straordinario passaggio delle sue Observationes medicae che merita di essere riportato:

... in qualunque modo ci si possa impegnare per scoprire come opera la Natura ... questa rimarrà sempre sconosciuta all'uomo. Non c'è da meravigliarsi. É infinitamente più credibile il fatto che noi, esseri miserabili, vagabondi lontani dal luminoso cammino della conoscenza, ci troviamo incapaci di comprendere il metodo dell'Artefice Supremo nella sua splendida e saggia opera, come un volgare fabbro che sia niente più che un rude ammiratore della maestria squisitamente elegante di un orologio ${ }^{63}$.

In effetti, c'è da ricordare che Sydenham fu anche il puritano autore della Theologia rationalis. Il rifiuto della pretesa di conoscere la natura aveva dunque una ragione non solo epistemologica, ma anche morale e teologica. Nella Prefazione curata da Locke alle Observationes medicae di Sydenham, i due amici e autori empiristi espressero una conclusione che, a mio avviso, tanta importanza ebbe anche nel successivo sviluppo della stessa medicina neoterica:

Scrivere la storia naturale di ciascuna malattia, scartando rigorosamente tutte le ipotesi, ecco l'unico mezzo di scoprirne le cause, se non quelle remote e segrete (speranza chimerica), almeno le cause prossime e immediate, che noi possiamo riconoscere e che ci indicheranno le terapie $^{64}$.

61 J. F. Payne, Thomas Sydenham, London, T. Fisher Unwin, 1900, 190.

62 D. E. Wolfe, Sydenham and Locke on the Limits of Anatomy, cit., 209.

63 T. Sydenham, Observationes medicae circa morborum acutorum historiam et curationem, Londini, Typis A. C. Impensis Gualteri Kettilby, ad insigne Capitis Episcopalis in Coemeterio D. Pauli, 1676, 171.

64 T. Sydenham, Observationes medicae, cit., p. 3. Si vedano anche H. Marion, J. Locke. Sa vie et son ouvre d'apres des documents nouveaux, Paris, Librarie GermerBaillière et Cie, 1878, 102, e P. Rossi, Ragionamento causale e ragionamento teleologico in medicina. La disputa fra medicina dogmatica, empirica e razionale nel pensiero di 
Il rischio d'uno sbilanciamento verso la teoria e d'un conseguente allontanamento dalla realtà empirica dei fenomeni costituiva una caratteristica della medicina neoterica chiaramente percepita non solo dai medici stessi e dagli addetti ai lavori. Il poeta e drammaturgo inglese Thomas Shadwell (c. 1642-1692), per esempio, scrisse una commedia che ironizzava proprio su quest'aspetto, intitolata Virtuoso, termine col quale si identificavano gli sperimentatori moderni, e rivolta, in questo caso, a quelli della Royal Society. La commedia fu rappresentata la prima volta nel 1676. In una scena, Sir Nicholas Gimrack, caricatura del virtuoso, era raffigurato nel tentativo di imparare a nuotare. Lo si vedeva osservare i movimenti d'una rana, imitarli disteso su d'un tavolo, ma senza infine mettere in pratica i gesti appresi. Dichiarava infatti: «Mi accontento della parte speculativa del nuoto. Non m'interessa la pratica. Raramente metto in pratica qualsiasi cosa, non è nel mio stile. La conoscenza è il mio ultimo fine» ${ }^{65}$. Sebbene la commedia ironizzasse sui 'virtuosi' in genere, è evidente che riguardasse anche la medicina. Il fatto che i medici neoterici spesso si basassero su conoscenze prive di una immediata ricaduta e utilità pratica, cioè in grado di migliorare la terapia, rappresentava un fattore che si esponeva facilmente a questo genere di critiche (sul quale torneremo).

Oltre all'anatomia microscopica, anche l'eccessivo basarsi della medicina sull'anatomia macroscopica costitutiva un aspetto della medicina neoterica fortemente criticato dagli esponenti d'una medicina empirica maggiormente focalizzata sulle manifestazioni cliniche delle malattie. Per esempio, il medico inglese Gideon Harvey (1640-1700 circa) (in nessuna relazione con l'Harvey scopritore della circolazione del sangue), nel 1683 pubblicò The conclave of physicians, detecting their intrigues, fraud, and plots, against their patients nel quale paragonava l'anatomia nientemeno che alla pratica del cannibalismo. Il 'conclave' dei medici, in stretta analogia a quello cattolico, era composto da un medico-papa e da diversi medici-cardinali, ritenuti infallibili perché fondati su una religione-medica rivelata. Nell'Introduzione del Conclave, dunque, Harvey si esprimeva come segue:

Marcello Malpighi, in E. Agazzi e C. Viesca (a cura di), Le cause della malattia. Un'analisi storica e concettuale, Genova, Erga edizioni, 1999, 49.

65 Citato in L. S. King, The philosophy of medicine. The early eighteenth century, Cambridge, MA/London, Harvard University Press, 1978, 87. 
Le loro immolazioni sono celebrate soprattutto d'inverno su cani e gatti dal più giovane, o talvolta sui corpi umani dal boia, loro servile funzionario, che li lascia poi ai cardinali dell'arte che intagliano e uccidono queste carcasse come cannibali. A questo scopo tutti gli spettatori (ai quali sono garantite libera entrata e uscita) possono osservare ... i medici che pretendono di eseguire ricerche più specifiche nelle parti del corpo umano, insinuando in essi la consapevolezza delle loro abilità e conoscenze incomparabili, non senza una semplice allusione al fatto che possano rivolgersi a loro in caso di malattia ${ }^{66}$.

Harvey, quindi, concludeva amaramente che l'idea dell'anatomia come strumento del progresso medico fosse un'illusione creata solamente per attrarre una maggiore clientela. Gli anatomisti, infatti, ostentavano di fronte al pubblico solo corpi morti, «corpi sicuri» perché non più curabili, piuttosto che mettere in mostra pazienti vivi e doloranti. Non c'era perciò dubbio sul fatto che avessero tanto successo nel promuovere sé stessi. Harvey, infine, sosteneva che le loro scoperte anatomiche non avessero alcun valore nella pratica:

E quante scoperte hanno fatto in Anatomia ...? Certamente nessuna, e io stesso oso presumere di sostenere d'aver divulgato osservazioni anatomiche più utili di tutti loro messi insieme; e inoltre devo confessare che se avessi impiegato le ore trascorse negli esercizi anatomici in altre parti della medicina, sarei ora un medico migliore ${ }^{67}$.

Questa stessa critica sarà utilizzata, come vedremo fra poco, anche dagli avversari di Malpighi in Italia.

\section{MALPIgHi, MORGAGNi E IL DIBATTITO FRA MEDICINA RAZIONALE E MEDICINA EMPIRICA}

Le opere di Sydenham, sebbene mai citate direttamente, furono con ogni probabilità tema di ispirazione per Giovanni Girolamo Sbaraglia (1641-1710), collega di Malpighi nello Studio di Bologna, che fra 1689 e 1704, cioè lungo ben 15 anni, scrisse una serie di 4 opuscoli,

66 G. Harvey, The conclave of physicians, detecting their intrigues, fraud, and plots, against their patients, London, Printed for James Partridge, 1683, 10-11.

67 G. Harvey, The conclave of physicians, cit., 12-13. 
i primi due in forma anonima, poi pubblicamente riconosciuti, in cui attaccava aspramente Malpighi sia da un punto di vista personale sia, ciò che più ci interessa, dal punto di vista della sua medicina neoterica. Significativamente, Sbaraglia era un docente di anatomia, cioè di quella stessa disciplina al centro della sua critica contro Malpighi ${ }^{68}$. E sappiamo persino che, durante le sue lezioni universitarie, Sbaraglia dimostrasse di essere piuttosto vicino ai moderni sia per metodo d'insegnamento sia per i contenuti. Antonio Vallisneri, che fu suo allievo, ci ha infatti trasmesso gli appunti presi nel corso delle sue lezioni nei bienni 1683-1684 e 1684-1685 e che oggi si trovano conservati presso la Biblioteca dell'Università di Padova ${ }^{69}$. Ebbene, da queste lezioni si ricava che Sbaraglia si basasse non solo sul commento degli autori antichi, ma anche su teorie e interpretazioni derivate dalle proprie ricerche e su resoconti di esperimenti per confutare le opinioni altrui. Coinvolgeva gli studenti, inoltre, nell'esecuzione di semplici esperimenti, insegnando loro come questi potessero essere interpretati ${ }^{70}$. Infine, pur difendendo a spada tratta l'antica teoria degli umori, dei temperamenti e degli spiriti, sosteneva che, in particolare, la dottrina degli spiriti contenesse ambiguità e paralogismi. Per esempio, la teoria galenica dell'origine dello spirito naturale nel fegato era scorretta, perché le ricerche moderne di Malpighi e Bellini avevano dimostrato che fosse un organo costruito soltanto per filtrare le impurità del sangue ${ }^{71}$.

$\mathrm{Ma}$ allora, ci si potrebbe chiedere, perché Sbaraglia attaccò Malpighi e la medicina moderna? Sintetizzando, due sono i motivi principali, ciascuno dei quali fu sviluppato a fondo nella prima e nell'ultima delle sue opere polemiche. La prima, pubblicata in forma anonima nella primavera del 1689 e intitolata De recentiorum medicorum studio dissertatio epistolaris ad amicum, è descritta da Malpighi come segue: «In questo libretto l'autore, abbracciata la setta empirica, tenta di condannare gli esercizi di anatomia sottile condotti col microscopio sull'uomo, sugli

68 Per ironia della sorte, le statue di Malpighi e Sbaraglia, ancora oggi, si trovano proprio una a fianco all'altra nel celebre teatro anatomico bolognese risalente al 1637.

69 F. Zampieri, Il metodo anatomo-clinico fra meccanicismo ed empirismo, cit., 118.

70 B. Dooley, La scienza in aula nella rivoluzione scientifica: dallo Sbaraglia al Vallisneri, Quaderni per la storia dell'Università di Padova 21 (1988), 23-41, qui 27-29.

71 B. Dooley, La scienza in aula nella rivoluzione scientifica, cit., 32. 
animali e sulle piante; li censura e li ridicolizza come aborti dell'ingegno inutili e libidinosi $>^{72}$. In tale scritto Sbaraglia, sostanzialmente, cercava di dimostrare che l'anatomia microscopica, insieme all'anatomia comparata e all'anatomia delle piante, fosse una scienza inutile per la medicina pratica, cioè per la clinica e per la cura delle malattie. L'unico mezzo per essere buoni medici era quello di esaminare le malattie nelle loro cause e manifestazioni più distinte e chiaramente osservabili. In effetti, a quel tempo, era in un certo modo innegabile il fatto che le nuove scoperte dei medici razionali non avessero portato a significative rivoluzioni in ambito terapeutico. Sbaraglia, quindi, non poteva che ribadire che solo l'«experientia» e l'«observatione» dovessero condurre l'azione del medico pratico nel somministrare i farmaci e nel curare le malattie, non le speculazioni e le nuove scoperte dei «neoterici» ${ }^{73}$.

Nell'ultimo dei suoi opuscoli, invece, intitolato Oculorum et mentis vigiliae, Sbaraglia sosteneva che il meccanicismo fosse inadeguato alla spiegazione dei fenomeni vitali, in quanto questi venivano attivati da un' anima che non seguiva, in quanto tale, le leggi geometriche e matematiche applicabili alla materia inanimata ${ }^{74}$. In questo caso, dunque, il medico bolognese sosteneva una tesi di matrice vitalistica. Probabilmente si trattava di idee che circolavano in ambito internazionale da qualche tempo, visto che, qualche anno dopo, il medico tedesco Georg Ernst Stahl (1659-1734), nella Theoria medica vera del 1708 , sviluppava la teoria secondo la quale il corpo fosse controllato da un'anima che dava forza e direzione, teleologicamente orientata, ai processi organici. Stahl, tra l'altro, aveva come riferimenti critici soprattutto gli approcci materialistici e meccanicistici di Boerhaave e di Hoffmann e il suo vitalismo costituiva una prima sistematica messa

72 M. Malpighi, Marcelli Malpighi opera posthuma, 2 voll., Londini, Impensis A. \& J. Churchill, ad insigne nigri cygni in vico dicto Pater-noster-Row, 1697, vol. 1, 102.

73 G. Sbaraglia, De recentiorum medicorum studio dissertatio epistolaris ad amicum (1689), in M. Malpighi, Marcelli Malpighi opera posthuma, cit., vol. 2, 84-91, qui 90.

74 G. Sbaraglia, Oculorum et mentis vigiliae, ad distinguendum studium anatomicum et ad praxim medicam dirigendam. Accedit mantissa subsidiaria de vi indicationis a parte et de usu microscopii, Bononiae, Typis Petri Mariae Monti, 1704, 251-254.

75 G. E. Stahl, Theoria medica vera. Physiologiam \& pathologiam, tanquam doctrinae medicae partes vere contemplativas, naturae $\mathcal{E}$ artis veris fundamentis, intaminata ratione, E inconcussa experientia sistens, Halae, Literis Orphanotrophei, 1708. 
in discussione di questa tipologia di medicina ${ }^{75}$. Stahl era convinto che la nuda materia fosse pura passività e che il suo meccanismo non potesse in alcun modo spiegare la complessità dei fenomeni e delle reazioni che avvenivano nei corpi viventi. La teoria corpuscolare dei fluidi non era sufficiente a spiegarne il comportamento fisiologico, il quale rivelava a ogni passo una finalità che rendeva imprescindibile il ricorso a un'anima che fornisse precisi orientamenti alle particelle. Per esempio, che i corpuscoli di dimensione diversa fossero filtrati da ghiandole diverse, sulla base della specifica tessitura dei loro filtri, non spiegava come mai le particelle più piccole non trapelassero attraverso filtri costruiti per quelle più grandi ${ }^{76}$. Era necessaria, quindi, un'anima che dirigesse costantemente i processi altrimenti meccanici del corpo.

Sbaraglia ebbe quindi buon gioco nel sollevare dubbi sull'efficacia della medicina neoterica, sia perché si poggiava su riserve condivise a livello internazionale - alcune delle quali ammesse dagli stessi medici neoterici - sia perché tali riserve avevano un certo fondo di verità. Per quanto espresse in modo tendenzioso - come vedremo fra breve - le critiche di Sbaraglia erano perciò fondate e non derivavano, perlomeno non esclusivamente, da giudizi dettati da questioni personali, o da una sorta di provincialismo culturale.

Sin dalla pubblicazione del primo opuscolo di Sbaraglia si scatenò, in Italia e in Europa, una disputa feroce basata su di una lunghissima serie di lettere, risposte, contro-risposte, opuscoli, opere firmate, anonime e alcune persino coperte da pseudonimi, che si protrasse fino alla seconda metà del Settecento. Malpighi, innanzitutto, scrisse una lunga replica, circolata manoscritta e, infine, pubblicata postuma, in cui ribatteva punto per punto alle tesi dello Sbaraglia ${ }^{77}$. Per il tenore epistemologico dello scritto, può essere considerata come un vero e proprio «testamento scientifico» ${ }^{78}$. La prima lettera di

76 F. P. De Ceglia, I fari di Halle, cit., 177.

77 M. Malpighi, Risposta del Dottor Marcello Malpighi alla lettera intitolata De recentiorum medicorum, cit.

78 L. Belloni (a cura di), Opere scelte di Marcello Malpighi, Torino, Unione Tipografico-Editrice Torinese, 1967, 443. Anche Howard Adelmann, nella sua monumentale opera sull'embriologia in Malpighi, sosteneva un'idea simile: «La sua importanza è legata al fatto che, insieme alla sua autobiografia, costituisce l'apologia che Malpighi, quasi al termine della sua vita, si sentì obbligato a scrivere sul suo lavoro, uno specchio 
Sbaraglia fu criticata anche da Giovanni Paolo Ferrari di Parma con un testo del 1690 intitolato Zelotypia veritatis in veterum fallaciis $\mathcal{E}$ dogmatibus, e da Johannes Bohn (1640-1718) di Lipsia con una dissertazione al principio delle sue Praelectiones therapenticas del $1691^{79}$. Sbaraglia non si curò delle critiche di Ferrari, mentre per rispondere a Bohn pubblicò una seconda lettera, sempre anonima, nel 1693, intitolata De recentiorum medicorum studio, dissertatio epistolaris secunda ad amicum, dove ripubblicò anche la prima ${ }^{80}$. In seguito alla pubblicazione dell'opera postuma di Malpighi, Sbaraglia riconobbe ufficialmente la paternità delle due opere ${ }^{81}$. Entrambe le lettere furono ulteriormente ristampate da Sbaraglia nelle sue Exercitationes physicoanatomicae, unitamente a un'appendice dal titolo Ad physico-anatomicas epistolares dissertationes appendix ${ }^{82}$. Sbaraglia, infine, scrisse il suo ultimo testo, il più lungo ed elaborato di tutti, cioè il già citato Oculorum, et mentis vigiliae.

Proprio con l'uscita di quest'ultimo scritto entra in scena anche Morgagni. All'epoca, Morgagni si era da poco laureato in medicina e filosofia a Bologna, essendo stato discepolo prediletto, come già accennato, di Valsalva. Tra l'altro, alla discussione della tesi, era presente lo stesso Sbaraglia, che non si esimette dal lodare il giovane studente. Nel 1704, anno della pubblicazione dell'Oculorum, Morgagni era stato eletto «Principe» dell'Accademia degli Inquieti di Bologna che, come già accennato, faceva parte di quel circuito di accademie sorte in epoca moderna in tutt'Europa che si facevano promotrici di nuove ricerche sperimentali. E Morgagni s'impegnò a fondo nella nuova carica, promuovendo un rinnovamento dell'Accademia sulla base dei più recenti modelli internazionali ${ }^{83}$. Secondo quanto scrive lo stesso Morgagni, fu proprio questo ruolo a imporgli, in un certo modo, di schierarsi a difesa

non solo del suo pensiero maturo, ma anche del suo stesso carattere»: H. B. Adelmann, Marcello Malpighi and the evolution of embriology, 5 vols, New York, Cornell University Press, 1966, vol. 1, 587.

79 A. Zeno, Relazione della controversia dello Sbaraglia col Malpighi, e tra i loro seguaci, Giornale de' letterati d'Italia 4 (1710), 262-292, qui 271.

80 A. Zeno, Relazione della controversia dello Sbaraglia col Malpighi, cit., 270.

81 A. Zeno, Relazione della controversia dello Sbaraglia col Malpighi, cit., 271.

82 G. Sbaraglia, Exercitationes physico-anatomicae, Bononiae, Typis Petri Mariae Monti, 1701.

83 B. Dooley, La scienza in aula nella rivoluzione scientifica, cit., 17. 
di Malpighi. Infatti, nel 1705 furono pubblicate, a Roma, due lettere firmate con gli pseudonimi di Luca Terranova e Orazio Floriani, ma opera proprio di Morgagni ${ }^{84}$. Nella sua autobiografia, Morgagni racconta la vicenda con queste parole:

Nel mentre, per via di quell'Accademia a lui affidata, il Morgagni opponeva ai colpi mancini la sua fermezza d'animo, ad altri invece andava incontro per amore di chi era stato il comune Maestro di ciascuno dei propri maestri, cioè M. Malpighi. Costoro lo andavano pregando di prendere la difesa di lui, Malpighi, messo sotto accusa nelle Vigiliae dallo Sbaraglia. Resosi ben conto della giustizia della causa e non solo per questo ma anche per un senso di gratitudine, egli acconsenti ${ }^{85}$.

Fu proprio grazie alla scoperta delle autobiografie di Morgagni, tra l'altro, che si ebbe la definitiva conferma che l'autore di queste lettere fosse stato proprio il forlivese. E questa non fu la sola volta che Morgagni prese direttamente parte alla polemica. In seguito alla pubblicazione anonima del De moralibus criticae regulis compendiosa monita nel $1706^{86}$ - testo che difendeva Sbaraglia contro Malpighi e contro le lettere di Morgagni - lo stesso Morgagni scrisse una Disputatio critico-moralis che circolò manoscritta nell'ambiente veneziano e padovano ${ }^{87}$. Morgagni, infatti, aveva già lasciato Bologna e si trovava, in quel periodo, a Venezia per approfondire i suoi studi e per intrattenere relazioni utili per la sua chiamata all'Università di Padova. Di questa dissertazione abbiamo un resoconto da parte dello scrittore veneziano

84 G.B. Morgagni, Horatii de Florianis M.E.P. epistola, qua plus centum, E quinquaginta errores ostenduntur in recenti libro inscripto oculorum, $E$ mentis vigiliae ad distinguendum studium anatomicum, $\mathcal{E}$ ad praxim medicam dirigendam: nec non inclyti viri, philosophi, medici, E anatomicorum nostrae aetatis facile Principis Marcelli Malpighi innumeri loci propugnantur, E exponuntur. Huic praefixa est quasi vice praefationis altera epistola in illud idem argumentum a Luca Terranova, Romae, s.e., 1705.

85 G.B. Morgagni, Opera postuma (Ms. Laurenziano Fondo Asbburnhamiano 227-159). I: Le autobiografie, Roma, Istituto di Storia della Medicina dell'Università di Roma, 1964, 15.

86 Anon., De moralibus criticae regulis compendiosa monita, ad quorum norman veluti obier exiguntur tum controversia prius agitata inter celeberrimos viros Marcellum Malpighium, et Joannem Hieronymum Sbaraleam, tum quedam epistolae nuper a quibusdam illius asseclis adversùs bunc evulgatae, Coloniae, Typis Eymerici de Francal, 1706.

87 G. Ongaro, Morgagni, Giovanni Battista, in Dizionario Biografico degli Italiani, Roma, Istituto della Enciclopedia Italiana, 2012, vol. 76, 744. 
Apostolo Zeno (1668-1750), apparso nel Giornale de' letterati d'Italia, dove emergeva il seguente giudizio:

In questo Manoscritto (di pagg. 118. in 4.) di cui non abbiam potuto saper l'Autore, trovammo uno stile così elegante, e sì ben meschiato il grave al faceto, che più d'una volta lo rileggemmo; ma vi trovammo particolarmente renduta la pariglia all'Autor delle Regole, e la materia maneggiata non solo con fondamentio, e copia di ragioni, ma eziandio con somma ricchezza, e facilità ${ }^{88}$.

La lettera di Terranova aveva un tono spiccatamente morale, in quanto tendeva a dimostrare che Sbaraglia, nelle sue opere contro Malpighi, si fosse dimostrato «altero», «falso», «sprezzante degli altri», «scarsamente erudito» e dallo «stile difettoso» ${ }^{89}$. Allo stesso modo, nella Disputatio critico-moralis del 1707, Morgagni si preoccupava soprattutto di documentare la malafede di Sbaraglia, piuttosto che difendere direttamente e radicalmente il metodo e i risultati, soprattutto teorici, di Malpighi. Nel recensire la Disputatio, Apostolo Zeno scrive:

... che la prima Lettera dello Sbaraglia sia calunniosa, egli è palese ad ognuno, perché ognuno sa, studiarsi da' Medici moderni, e doversi infatti, come da' Medici onorati studiare tante altre cose oltre alle tre Notomie; e pure in queste dà ad intendere lo Sbaraglia, che consistano gli studj loro, e per conseguenza li calunnia .... ${ }^{90}$

Molto significativo, in ogni caso, il fatto che Morgagni sottolineasse con forza un aspetto sostenuto dallo stesso Malpighi, cioè che la medicina dei moderni non si limitasse solo alle tre anatomie, cioè microscopica, comparata e delle piante, come pretendeva Sbaraglia, ma si rivolgesse anche a molte altre cose proprie dell'indagine medica. Risulta come se Morgagni volesse ridimensionare, in fondo, l'importanza dell'interpretazione dell'anatomia tramite rigidi modelli meccanicistici, poiché la ricerca anatomica non era mai fine a sé stessa, ma restava sempre rivolta alla comprensione e alla cura delle malattie.

La lettera di Floriani, invece, sviluppava un contenuto maggiormente epistemologico, rivelandosi quindi particolarmente significativa

88 A. Zeno, Relazione della controversia dello Sbaraglia col Malpighi, cit., 283.

89 A. Zeno, Relazione della controversia dello Sbaraglia col Malpighi, cit., 278.

90 A. Zeno, Relazione della controversia dello Sbaraglia col Malpighi, cit., 287. 
in relazione alla concezione metodologica che Morgagni avrebbe maturato nelle sue opere successive. Marta Cavazza, in un'analisi della lettera di Floriani, sosteneva quanto come segue:

Le difficoltà concettuali e pratiche, oltre che ideologiche, dell'automatismo e delle teorie iatromeccaniche in generale, come pure della medicina 'razionale' ad esse direttamente collegata, erano comunque reali e all'inizio del Settecento anche i continuatori della tradizione meccanicista cominciavano a prenderne atto. Il tono di maggior cautela delle Epistolae rispetto alla Risposta malpighiana potrebbe essere indicativo di una certa presa di distanza, ovviamente implicita, dalle formulazioni più decise e coerenti del meccanicismo psico-biologico, la difesa del quale è piuttosto d'ufficio... Rispetto a quest'ultimo, Morgagni sembra ben più incerto sulla possibilità e l'estensione dell'applicazione della matematica alla medicina e sembra quasi più preoccupato di stabilire i limiti piuttosto che la legittimità del ricorso alla deduzione a priori da parte del medico ${ }^{91}$.

L'aspetto più interessante, per quanto riguarda il punto di vista metodologico, era che, nella lettera di Floriani, Morgagni avesse avanzato alcune cautele circa l'applicazione del ragionamento a priori in medicina che, nei termini malpighiani, significava basare l'argomentazione medica su modelli teorico-meccanici di struttura e funzionamento del corpo umano, per poi applicare le deduzioni rese possibili dai modelli alla realtà fisiologica e fisiopatologica. Non a caso, Morgagni si preoccupava d'accusare Sbaraglia di malafede, perché interpretava le espressioni di Malpighi, radicalizzandole, come se egli intendesse dire che in medicina si potessero dedurre verità in modo dimostrativo sulla base d'una conoscenza precostituita delle cause e dei principi ultimi:

[...] [Sbaraglia] ha inteso tutti quei luoghi non secondo il modo di esprimersi dei medici, ma come circoscritte proposizioni di un matematico molto rigido. E tuttavia se queste parole sono quelle che egli ha inteso spesso e malamente con un ragionamento difficilissimo e per nulla sostenibile, esse sono quelle che sono state impiegate spesso dal tuo maestro [Malpighi], decisamente con un altro senso, nella sua Risposta, cioè procedere a priori, predire a priori, dedurre a priori, etc.

91 M. Cavazza, L'impegno del giovane Morgagni per la riforma dell'Accademia degli Inquieti e in difesa della tradizione malpighiana, in V. Cappelletti e F. Di Trocchio (a cura di), De sedibus, et causis. Morgagni nel centenario, cit., 91-103, qui 100-101. 
che secondo i medici razionali indicano certamente "progresso", "predizione", "deduzione" attraverso l'aiuto del ragionamento e la considerazione delle cause, dato che vengono distinte da quelle che gli empirici ignoranti stabiliscono e considerano soltanto in base alla cieca esperienza. Infatti, come negli Opuscoli, così in tutto questo libro delle Vigilie il nostro [Sbaraglia] chiarisce così quelle parole, quasi che dedurre a priori sia stato detto senza dubbio per intendere "dedurre in modo dimostrativo e con la conoscenza di tutte le cause fin dai primi principi, etc.”. Per cui accade poi che egli senza fatica (tutti potrebbero farlo!) mostra mille affermazioni assurde di un autore mal interpretato, certamente contraddizioni, cattive illazioni e soprattutto una grandissima incoerenza, mentre quell'Uomo (come egli dice), che aveva dedotto ogni cosa sempre in modo probabile, d'improvviso in questa sua Risposta osa dedurre ogni cosa in modo dimostrativo ${ }^{92}$.

Malpighi, dunque, aveva «dedotto ogni cosa sempre in modo probabile». A riprova che Malpighi non utilizzasse un criterio rigidamente dimostrativo, Morgagni faceva notare che il suo metodo non si discostasse da quello utilizzato persino da Ippocrate:

E certamente quando il tuo maestro [Malpighi] dice a p. 353 della medesima Risposta che Ippocrate registrò storie particolari che servono da esempio e da induzione, o almeno da dimostrazione a posteriori di ciò che, procedendo a priori, spiegò in altri suoi libri di medicina, chi potrebbe sostenere che il tuo maestro abbia creduto che in tali libri Ippocrate procedesse in modo dimostrativo? ?3 $^{93}$

Che fosse la verità o meno riguardo all'approccio malpighiano, Morgagni avrebbe seguito un approccio iatromeccanico molto più cauto nel prosieguo di tutta la sua carriera, come testimoniato dalla natura delle sue opere che, proprio sulla base di questo contesto polemico, possono essere comprese appieno. Una cautela, tra l'altro, probabilmente suggerita non solo da questioni squisitamente scientifiche, ma anche personali, viste le difficoltà che Morgagni incontrò sia in quanto appartenente alla scuola di Malpighi sia in quanto sospettato autore delle epistole del 1705. Come sappiamo, Morgagni fu in un certo modo costretto a lasciare Bologna a causa delle gelosie suscitate dal suo rapporto con Valsalva, in particolare dopo che Morgagni lo sostituì nell'a-

92 G. B. Morgagni, Horatii de Florianis M.E.P. epistola, cit., 83.

93 G. B. Morgagni, Horatii de Florianis M.E.P. epistola, cit., 84. 
natomia pubblica del 1706, mentre Valsalva era a Parma, perché si pensò che fosse un passo decisivo verso la sua promozione alla lettura d'Anatomia proprio al posto del Maestro. Morgagni, inoltre, temette persino per la propria incolumità personale a causa dei sempre maggiori sospetti riguardo la paternità delle lettere pubblicate a Roma, tant'è che il 27 dicembre 1705 vergò il primo dei suoi testamenti94. Tali lettere avevano suscitato tanta rabbia in Sbaraglia da portarlo a promettere una ricompensa in denaro a chi ne avesse smascherato l'autore ${ }^{95}$. «L'Alma mater era diventata matrigna», nell'incisiva definizione di Giuseppe Ongaro".

Ebbene, sin dalla sua prima opera anatomica, gli Adversaria anatomica prima che lo consacrò come fra i più promettenti anatomisti d'Europa ${ }^{97}$, Morgagni dimostrava una certa presa di distanza perlomeno dalle versioni più radicali dell'approccio di Malpighi. Per quanto tale lavoro fosse, per certi versi, un manifesto dell'anatomia sottile malpighiana e per quanto, sul modello del maestro, Morgagni spesso si focalizzasse sull'individuazione e descrizione delle ghiandole nei tessuti investigati, non vi è traccia, innanzitutto, dell'uso del microscopio, cioè proprio dello strumento principale che permise a Malpighi di realizzare le sue scoperte maggiori, così come Morgagni non lo utilizzerà mai nelle sue opere successive. Egli, infatti, non era convinto dell'affidabilità di questo strumento, perché poteva indurre a osservare 'artefatti' che non corrispondevano alla reale struttura anatomica del corpo ${ }^{98}$. Il fatto che il microscopio fosse uno strumento tanto promettente quanto fuorviante costituì una riserva piuttosto comune a coloro che diffidavano della

94 F. Aulizio, L'inedito testamento di Giambattista Morgagni redatto il 27 dicembre 1705 in Bologna, Pagine di storia della medicina 8 (1964), 2-13.

95 F. Zampieri, Il metodo anatomo-clinico fra meccanicismo ed empirismo, cit., 383.

96 G. Ongaro, Morgagni a Bologna, in L. Rossetti (a cura di), Rapporti fra le università di Padova e Bologna: ricerche di filosofia, medicina e scienza, Trieste, Edizioni Lint, 1988, 255-306, qui 284. Pisarri, 1706.

97 G. B. Morgagni, Adversaria anatomica prima, Bononiae, typis Ferdinandi

98 Giuseppe Mosca, per esempio, nella prima biografia ufficiale su Morgagni, scritta quando lui era ancora in vita, scriveva che «[avesse] un lodevol timore degl'inganni de' Microscopj ... onde volle più tosto interrogar la Natura nella sua libertà»: G. Mosca, Vita di Giovambattista Morgagni, Napoli, presso Gennaro Migliaccio, 1768,34 . 
medicina neoterica, in Italia come nel resto d'Europa. Il medico e letterato fiorentino Anton Francesco Bertini (1658-1726), per esempio, nel 1699 scrisse la Medicina difesa dalle calunnie degli uomini volgari, e dalle opposizioni de' dotti che suscitò le consuete polemiche - attraverso scambi epistolari, discussioni pubbliche, opere a stampa d'accusa e di difesa - per questo genere di lavori ${ }^{99}$. A proposito del microscopio, avanzava le stesse critiche proposte da Sbaraglia, fondate sul fatto che tale strumento rivelasse immagini a volte di difficile interpretazione che, perciò, potevano confondere le idee, piuttosto che permettere la scoperta di qualcosa di nuovo ${ }^{100}$. Inoltre, sia in questi primi Adversaria sia in tutte le altre opere anatomiche successive, Morgagni avrebbe costantemente dimostrato un profondo legame con la letteratura sia antica che moderna. Ogni descrizione anatomica, cioè, doveva essere corroborata da una sistematica analisi delle fonti come verifica e confronto degli eventuali nuovi dati acquisiti. In questo modo, non vi era solo medicina neoterica, ma si manteneva anche un costante confronto con gli antichi. Basti pensare all'anonima recensione degli Adversaria apparsa nel secondo volume del Giornale de' letterati d'Italia del 1710, dove si sottolineava la capacità di Morgagni di rivitalizzare la scienza classica, recuperando scoperte anatomiche del passato dimenticate, da lui confermate e arricchite attraverso abili ricerche al tavolo anatomico ${ }^{101}$.

Per quanto riguarda la sua seconda opera a stampa, il già citato Nova institutionum medicarum idea - in cui Morgagni, assumendo la cattedra di Medicina Teorica a Padova, delineava una riforma ideale del curriculum medico - se quel Nova poteva riecheggiare in qualche modo i medici neoterici, lo scritto era, allo stesso tempo, talmente immerso nella cultura classica e in perfetto equilibrio fra passato e presente da risultare innocuo anche per i professori padovani più conservatori. In una delle sue autobiografie, infatti, Morgagni si compiaceva del fatto che, dopo la lettura della sua Prolusione, «Grande e universale fu il plauso, e totale l'assenza di critiche: e si sa che su questo punto Padova

99 D. Celestino e M. Crespi, Bertini, Anton Francesco, in Dizionario Biografico degli Italiani, Roma, Istituto della Enciclopedia Italiana, 1967, vol. 9, 534-535, qui 535.

100 A. F. Bertini, La medicina difesa dalle calunnie degli uomini volgari, e dalle opposizioni de' dotti, Lucca, per i Marescandoli, 1699, 8.

101 Anon., Joannis Baptistae Morgagni Foroliviensis ... Adversaria anatomica prima ... di p. 48. di carta gr. con 4. Tavole in rame, Giornale de' letterati d'Italia 2 (1710), 222-240, 223. 
è una città difficilissima» ${ }^{102}$. Ricordiamo, brevemente, che anche Padova fu profondamente colpita dalla diatriba fra medici iatromeccanici e medici empirici. Vallisneri e Guglielmini vi presero parte e il primo, al principio della sua carriera patavina, fu particolarmente osteggiato, tanto da essere sul punto di smettere di insegnare teorie neoteriche ${ }^{103}$. Morgagni, dunque, ebbe un motivo in più per seguire una strada meno radicale sia nei metodi sia nel modo di presentare i risultati. Inoltre, in questa prolusione emergeva chiaramente la natura congetturale della medicina, quindi ben lungi dall'essere geometrico-dimostrativa come poteva risultare da certe applicazioni radicali dei modelli meccanici. Morgagni sosteneva che «le regole e i precetti» dell'arte medica non dovessero essere concepiti come assoluti: «... questi, infatti, non debbono essere riferiti come leggi immutabili e fisse, ma tali da essere soggette ai mutamenti suggeriti dall'esperienza, dall'applicazione, dalla riflessione e soprattutto dalla prudenza, a seconda del variare delle cause, dei tempi, dell'età e di altre circostanze del genere» ${ }^{104}$.

Parole molto diverse, queste, da quelle utilizzate da iatromeccanici di prima generazione come Malpighi e da Borelli. Il primo, per esempio, sosteneva che «... nelle cose, dunque, della natura, che opera per necessità sempre uniforme, la sagacità dell'uomo non è di così poca attività, che non possa arrivare a svelare buona parte dei suoi artifici ${ }^{105}$. Il secondo, nel suo De motu animalium, scriveva: «Poiché le operazioni della natura sono facili, semplici e in accordo con le leggi della meccanica, che sono le leggi della necessità ${ }^{106}$. L'uniformità e la costanza dei fenomeni naturali, in sostanza, sembravano essere alla base della possibilità di elaborarne una conoscenza certa.

$\mathrm{Al}$ fatto che la medicina fosse una scienza congetturale, Morgagni diede ampio spazio, sia nelle sue opere, sia nell'attività professionale e nelle lezioni universitarie. Per esempio, in un consulto medico datato 22 febbraio 1749, indirizzato a una signora di Crema a proposito della

102 G.B. Morgagni, Opera postuma (Ms. Laurenziano Fondo Asbburnbamiano 227-159). I: Le autobiografie, cit., 38.

103 Per una ricostruzione del dibattito padovano, si veda F. Zampieri, Il metodo anatomo-clinico fra meccanicismo ed empirismo, cit., 366-383.

104 G. B. Morgagni, Nova institutionum medicarum idea, cit., 40.

105 M. Malpighi, Risposta del Dottor Marcello Malpighi alla lettera intitolata De recentiorum medicorum, cit., 110.

106 G. A. Borelli, De motu animalium, cit., 73. 
sua guarigione, o meno, dal «morbo Gallico», Morgagni, ancor prima d'addentrarsi nella materia, scriveva: «Benché la Medicina sia una facoltà congetturale, e per conseguenza non possa, massimamente in certi casi, affermare, o negare con indubitata certezza; tuttavia ben ponderando le ragioni per l'una parte, e per l'altra, può decidere ciò che sia più credibile; sul qual fondamento saviamente in ogni materia congetturale suol regolarsi la Società Umana» ${ }^{107}$.

La questione veniva ripresa anche nelle lezioni di Medicina teorica focalizzate sul commento di Avicenna. Morgagni, dopo un lungo ragionamento ricco di citazioni classiche, sosteneva che la Medicina fosse sia una scienza sia un'arte: una scienza perché costituita di assiomi e dimostrazioni logiche, un'arte nella misura in cui questi assiomi si rivolgevano a circostanze sempre incerte e fluttuanti: «Così la facoltà fisico-matematica, quando si volge a circostanze incerte, è arte, ma prima di volgersi ad esse, è scienza, e tale è chiamata. Perciò la Medicina alla sua origine è scienza, e, quando si volge a circostanze incerte, tanto più diventa arte congetturale» ${ }^{108}$.

Infine, il suo De sedibus può essere considerato il risultato finale $\mathrm{e}$ il frutto più maturo di questo costante ripensamento dell'approccio iatromeccanico e della sua possibile relazione con una medicina più spiccatamente empirica. Come ben noto, il De sedibus è un'opera monumentale suddivisa in 5 libri, ciascuno dei quali dedicato a un settore corporeo: il primo per le malattie del capo; il secondo per quelle del torace; il terzo per quelle del ventre; il quarto per le malattie chirurgiche - quindi ferite, lussazioni e fratture - e quelle definite 'universali', che cioè colpivano tutto il corpo; il quinto, infine, era dedicato ad aggiunte ai quattro libri precedenti. Ogni libro era composto da diverse 'lettere anatomo-mediche', ciascuna delle quali dedicata allo studio di una specifica malattia. Ogni malattia, a sua volta, era indagata attraverso la descrizione e il confronto di molti casi individuali - in totale, ve ne sono circa 700 - ciascuno dei quali composto da una dettagliata storia clinica del paziente, di un minuzioso referto autoptico, e del tentativo di collegare i sintomi clinici

107 G. B. Morgagni, Consulti medici pubblicati da minute inedite, Bologna, L. Cappelli Editore, 1935, 358.

108 G. B. Morgagni, Opera Postuma (Ms. Laurenziano Fondo Ashburnbamiano 227-159). IV: Lezioni di medicina teorica: commento ad Avicenna, Roma, Istituto di Storia della Medicina dell'Università di Roma, 1969, 41. 
manifestati in vita con i danni organici rinvenuti nel cadavere. Già la vastità di questo tentativo dimostra un'idea più volte esplicitamente riportata da Morgagni: le malattie sono fenomeni complessi e ampiamente variabili da individuo a individuo. Per averne un quadro completo, dunque, era necessario analizzare il maggior numero possibile di casi individuali, di modo da poter almeno in parte padroneggiare l'infinità gamma di possibili variazioni. Il De sedibus è ricchissimo di passaggi in cui si sottolinea la complessità dei clinici e la conseguente impossibilità di costruire un modello concettuale unico che possa abbracciare tutte le possibili variazioni. Sin dalla Prefazione generale del De sedibus, egli sottolineava che erano «Rare ... le malattie, e quelle in ispecie di una certa durata, alle quali non se ne unisca qualche altra, o che da più e vari sintomi non vengano accompagnate $\rangle^{109}$. Questa complessità clinica era riconducibile a una complessità causale: «Certamente la malattia non è semplice come il suo nome, ma abbraccia numerose differenze prodotte da molte cause, non solo diverse, ma talvolta anche opposte» ${ }^{110}$. Di conseguenza, pochissime malattie, scriveva Morgagni, avevano il «... loro segno patognomico che le distingue, mentre la maggior parte sono il risultato di cause complesse che agiscono su più parti del corpo e che danno vita a una unione complessa di molti segni» ${ }^{111}$.

Per fare qualche esempio clinico, dopo aver presentato una serie di casi di «idropisia del pericardio», Morgagni concludeva: «Io volli dar qui un cenno di tutto questo all'oggetto di farti più agevolmente intendere che nel modo stesso che l'idropisia del pericardio può variare all'infinito, e per origine, e per la qualità e copia dell'acqua, così anche i di lei sintomi vanno soggetti a variazioni ... e soprattutto il polso» ${ }^{112}$. Allo stesso modo, dopo aver discusso a lungo di casi caratterizzati da «lamine ossee» rinvenute nelle coronarie - quindi di aterosclerosi Morgagni si esprimeva come segue:

... tuttavia, siccome ho spesso considerato sulla quantità delle differenze che per l'ordinario si offersero in ciò ch'è naturale, e molto più

109 G. B. Morgagni, De sedibus et causis morborum per anatomen indagatis, cit., Prefazione generale.

110 G. B. Morgagni, De sedibus et causis morborum per anatomen indagatis, cit., Prefazione al Libro Quarto.

111 G. B. Morgagni, De sedibus et causis morborum per anatomen indagatis, cit., Prefazione al Libro Secondo. 
ancora in ciò che non lo era, e siccome feci il confronto di tutte le mie osservazioni con quelle degli altri, così mi giova sperare che mi perdoneranno se di bel nuovo dirò che le cose hanno un andamento vario nei varj individui, ed in particolare riguardo a quanto appartiene a quelle concrezioni delle quali si è ora istituito l'esame ${ }^{113}$.

E sempre a proposito di «lamine ossee», Morgagni giungeva a una prudenziale sospensione di giudizio, in attesa che ulteriori ricerche potessero portare nuova luce a fenomeni ancora non del tutto compresi: «E questo basti; non già perché io voglia adottare un'opinione contraria, ma onde sospendere per un poco il mio assunto, in sino a tanto che la verità meglio si scuopra alle mie ricerche» ${ }^{114}$.

Questi elementi di variabilità e complessità così ben evidenziati nel De sedibus nei confronti delle malattie umane comportavano che Morgagni utilizzasse i modelli meccanicistici in un modo più cauto rispetto a molti altri rappresentanti di questa corrente. Il modello meccanico, cioè, risultava utile per ipotizzare i processi fisiopatologici che correlavano il danno organico con i sintomi clinici, ma, allo stesso tempo, mantenevano un valore euristico, cioè erano privati della pretesa di descrivere la malattia con precisione geometrico-matematica e in modo definito una volta per tutte. Per esempio, Morgagni ipotizzava che le lesioni alle arterie o alle ossa dell'infezione sifilitica fossero determinate dalla presenza di particelle «acuminate» nel sangue prodotte dalla malattia, ma si guardava bene da sostenere che questo modello corrispondesse alla realtà ${ }^{15}$. Anzi, Morgagni presentava questi suoi tentativi di spiegazione come «congetture» passibili di revisione o persino di radicale cambiamento attraverso, si badi bene, l'accumulo di nuove «osservazioni»:

Tu dunque intendesti i miei dubbi e le mie congetture, le quali non saranno da me abbandonate fino a che non si producano molte osser-

112 G. B. Morgagni, De sedibus et causis morborum per anatomen indagatis, cit., II, XVI, par. 44

113 G. B. Morgagni, De sedibus et causis morborum per anatomen indagatis, cit., II, XXVII, par. 27.

114 G. B. Morgagni, De sedibus et causis morborum per anatomen indagatis, cit., II, XVIII, par. 33.

115 F. Zampieri, A. Zanatta, C. Basso, e G. Thiene, Cardiovascular medicine in Morgagni's De sedibus: dawn of cardiovascular pathology, Cardiovascular patology 25 (2016), 443-452. 
vazioni, raccolte con somma accuratezza, che bene spesso ebbi a desiderare nelle storie, e che in fine mi conducano a riconoscere volentieri che ciò ch'io non impugno che possa ed abbia potuto accadere, sia in realtà accaduto ${ }^{116}$.

Un'altra caratteristica cruciale del De sedibus, forse non ancora sottolineata a sufficienza, consiste nel fatto che Morgagni dedicasse una grande attenzione non solo alla descrizione anatomica, punto focale della iatromeccanica, ma anche alle storie cliniche, nodo cruciale della medicina empirica. Non si trattava, quindi, di un lavoro di anatomia patologica per come la intendiamo oggi, ma, come lo definisce Morgagni stesso, di una «guida metodologica per il medico pratico» ${ }^{117}$. Davide Giordano (1864-1954), nella sua classica opera su Morgagni, definiva il De sedibus come «... il libro della anatomia clinica, piuttostoché, o solamente, patologica» ${ }^{118}$. Giuseppe Ongaro, nel più recente passato, faceva significativamente notare che il De sedibus fosse improntato secondo il punto di vista clinico, non anatomico, proprio perché le malattie, in quanto entità cliniche, costituivano gli oggetti principali della trattazione ${ }^{119}$. Pericle Di Pietro, allo stesso modo, sosteneva che «Il trattato morgagnano è pur sempre un'opera di medicina clinica, più che d'anatomia, anche se l'indagine al tavolo autoptico assurge a componente di rilievo» ${ }^{120}$. Lo psichiatra e neurologo Walther Riese (1890-1976), nella sua profonda analisi storico-epistemologica sul concetto di malattia, scriveva a proposito del De sedibus:

Che Morgagni, nonostante il suo interesse per l'anatomia patologica e i suoi contributi a questa scienza, non sconfessi il clinico, deriva proprio

116 G. B. Morgagni, De sedibus et causis morborum per anatomen indagatis, cit., II, XXIV, par. 23.

117 R.A. Bernabeo, Scienza e pratica dell'anatomia patologica dalle origini a Rudolph Virchow, Quaderni del raggruppamento Tosco-Umbro-Emiliano di storia della medicina 1 (1987), 31-43, qui 36.

118 D. Giordano, Morgagni, Torino, Unione Tipografico-Editrice Torinese, $1941,176$.

119 G. Ongaro, L'anatomia patologica e la clinica, in S. Petruccioli (a cura di), Storia della scienza, 10 voll., Roma, Istituto della Enciclopedia Italiana, 2001-2004, vol. VI (2002), 716-726, qui 722.

120 P. Di Pietro, L'opera di Giambattista Morgagni nell'ambito del pensiero medico-biologico del Settecento, in V. Cappelletti e F. Di Trocchio (a cura di), De sedibus, et causis. Morgagni nel centenario, cit., 47-51, qui 50. 
dalla maniera in cui egli ordinò il materiale. Ci si aspetterebbero delle suddivisioni secondo il principio regionale adottato; invece, il materiale di ogni libro fu raggruppato principalmente secondo i sintomi, cioè secondo le alterazioni funzionali (dolore, soffocazione, tosse, palpitazione, sincope, singhiozzo, vomito, paralisi, balbuzie, cecità, afonia, etc.), ma anche secondo le entità cliniche (apoplessia, delirio, melanconia, epilessia, itterizia, lue venerea, artrite, gotta, gonorrea, etc. $)^{121}$.

Era proprio la clinica, quindi, cioè la diagnosi e la cura delle malattie, l'interesse principale sviluppato da Morgagni con questo lavoro.

Infine, come già fatto anche nelle opere anatomiche, il ricorso alla letteratura sia antica che moderna - per rinvenire possibili confronti sia anatomici sia clinici con i casi osservati e descritti da Morgagni - era straordinariamente ricco e dettagliato. Per esempio, se Valsalva e Bonet erano, fra i moderni, gli autori maggiormente citati, fra gli antichi trovava amplissimo spazio - oltre, naturalmente, ai grandi classici come Ippocrate, Galeno e Celso - la figura di Areteo di Cappadocia, medico greco del I secolo d.C., che esercitò a Roma e fu autore d'un trattato sulle malattie in otto libri, suddivisi in quattro coppie, ciascuna delle quali intitolata: De causis et signis acutorum morborum, De causis et signis diuturnorum morborum, De curatione acutorum morborum e De curatione diuturnorum morborum. Nell'opera di Areteo ogni malattia era descritta, sistematicamente, secondo il sito anatomico, incluse osservazioni anatomo-patologiche; secondo il significato del nome; l'elenco e la descrizione dei sintomi; le cause in relazione, in particolare, all'età e al sesso del paziente, e alla stagione dell'anno ${ }^{122}$. Si trattava, dunque, di uno straordinario rappresentante di quell'anatomia medica di cui proprio Morgagni rivendicava l'antichità, e infatti il forlivese utilizzò ampiamente le sue descrizioni a confronto delle proprie.

Così come non vi era reale contrasto, in Morgagni, fra anatomia e clinica, fra iatromeccanica e medicina empirica, non vi era, dunque, alcun contrasto nemmeno fra medicina moderna e medicina antica, ma,

$121 \mathrm{~W}$. Riese, The conception of disease: its history, its versions and its nature, New York, Philosophical Library, 1953, 99.

122 L. Stroppiana, Areteo di Cappadocia. Le cause e $i$ sintomi delle malattie acute e croniche, Roma, De Luca, 1973. Vedi anche G. Weber, Areteo di Cappadocia. Interpretazioni e aspetti della formazione anatomo-patologica del Morgagni, Firenze, Leo S. Olschki, 1996, e F. Zampieri, Il metodo anatomo-clinico fra meccanicismo ed empirismo, cit., 203-205. 
anzi, una sostanziale e armoniosa continuità. Morgagni, in ultima analisi, seppe districarsi egregiamente in questa moltitudine di significati e possibili atteggiamenti nei confronti delle diverse 'sette' e tradizioni, sapendo sfruttare a suo vantaggio la fluidità delle distinzioni in gioco. Il successo del suo sforzo, però, non dipese solo da abilità dialettica, ma dal fatto sostanziale che il suo metodo anatomo-clinico costituì realmente una sintesi ragionata ed equilibrata fra meccanicismo ed empirismo, oltre che fra tradizione e innovazione. Ed è proprio per questo che, come sostenuto al principio di questo testo, si può sostenere che, con Morgagni, si chiude, in un certo modo, una tradizione millenaria di studi clinici e approcci anatomicamente orientati che, dopo essersi scontrati, incontrati e contaminati a vicenda, trovano un definitivo terreno d'intesa. Ricordiamo che non molti anni dopo la pubblicazione del De sedibus ci sarà la Rivoluzione francese che, oltre al rifiuto dell'ancien regime, portò al definitivo abbandono di qualunque modo precedente di fare e intendere la medicina. 
